\title{
The dynamical foundations of motion pattern formation: Stability, selective adaptation, and perceptual continuity
}

\author{
HOWARD S. HOCK \\ Florida Atlantic University, Boca Raton, Florida \\ GREGOR SCHÖNER \\ Ruhr-Universität Bochum, Bochum, Germany \\ and \\ MARTIN GIESE \\ Max Planck Institute for Biological Cybernetics, Tübingen, Germany
}

\begin{abstract}
A dynamical model is used to show that global motion pattern formation for several different apparent motion stimuli can be embodied in the stable distribution of activation over a population of concurrently activated, directionally selective motion detectors. The model, which is based on motion detectors being interactive, noisy, and self-stabilizing, accounts for such phenomena as bistability, spontaneous switching, hysteresis, and selective adaptation. Simulations show that dynamical solutions to the motion correspondence problem for a bistable stimulus (two qualitatively different patterns are formed) apply as well to the solution for a monostable stimulus (only one pattern is formed) and highlight the role of interactions among sequentially stimulated detectors in establishing the state dependence and, thereby, the temporal persistence of percepts.
\end{abstract}

In this article, an intuitively accessible dynamical model of motion pattern formation is proposed that accounts in a unified manner for bistability, spontaneous switching, hysteresis, and the effects of adaptation. The model is based on substantial evidence that motion detectors in the visual system are interactive (they mutually influence each other's activation), that the activation levels of neurons fluctuate randomly (Softky \& Koch, 1993), and most significantly, that mechanisms intrinsic to individual detectors stabilize their activation. On this basis, it is shown, for several different apparent motion stimuli, that perceived global motion patterns can be embodied in the stable distribution of activation over a population of concurrently activated, directionally selective motion detectors; higher order pattern detectors are not required to account for global pattern formation for these stimuli. The dynamical model shows that interactions between currently stimulated detectors and detectors that will be stimulated in the immediate future are crucial for establishing the temporal persistence of a perceived pattern (i.e., its temporal stability). It is demonstrated that the dynamical solution to the

Grant support from the Deutsche Volkswagenstiftung is gratefully acknowledged. We also thank David Nichols for his help with the simulations. Correspondence concerning this article should be addressed to H. S. Hock, Department of Psychology, Florida Atlantic University, Boca Raton, FL 33431 (e-mail: hockhs@fau.edu). motion correspondence problem for a bistable stimulus (for which two qualitatively different patterns are formed) applies as well to the solution for a monostable stimulus (for which only one pattern is formed), and new predictions emerge regarding solutions to the correspondence problem.

Our theoretical objective, therefore, is to unify traditional approaches, which have emphasized what is perceived when a motion stimulus is presented (i.e., the solution to the motion correspondence problem; see, e.g., Ullman, 1979), and dynamical approaches, which have emphasized the process of perceptual change (e.g., Ditzinger \& Haken, 1995; Kawamoto \& Anderson, 1985; Koechlin, Anton, \& Burnod, 1999; Schöner \& Hock, 1995). Although most of the same concepts are inherent in other dynamical models (e.g., Chey, Grossberg, \& Mingolla, 1997; Grossberg \& Mingolla, 1985; Grossberg \& Rudd, 1989; Koechlin et al., 1999; Williams, Phillips, \& Sekuler, 1986; Wilson, Ferrera, \& Yo, 1992), the proposed model stands apart for the following reasons. (1) It aims for theoretical parsimony and generalizability; the dynamical representation is derived from just a few very simple principles that apply irrespective of the particulars of the neurophysiological substrate (in contrast, e.g., with neurophysiologically specific mechanisms inherent in the dynamical models of such investigators as Grossberg, Mingolla, and colleagues or Koechlin et al.). (2) The function of activationstabilizing mechanisms is made explicit. (3) Interactions 
between simultaneously and sequentially stimulated detectors are distinguished with respect to their functional significance for pattern formation and pattern stability. (4) The dynamical representations are constrained by a wide range of dynamical data (measurements of spontaneous switching, hysteresis, and adaptation).(5) The framework is generalizable to motion pattern formation in other paradigms.

\section{IS STABILIZATION NECESSARY? EVIDENCE FROM BISTABLE STIMULI}

Natural, everyday percepts are very stable. It rarely happens that two qualitatively different percepts are formed for the same stimulus (bistability), and the experience of spontaneous switching between alternative percepts is likewise rare. Because everyday experiences of monostability are so pervasive, perceptual stability (and the potential for it to be lost) is not always recognized as an important problem for the perceptual psychologist. Historically, however, stability was once a central issue. William James (1890) famously recognized the problem of stable percepts emerging from the "blooming, buzzing confusion" of sensory input, and Wolfgang Köhler (1920/1938) theorized that perceptual experience was isomorphic to the stable, equilibrium states of electrical fields formed in the brain. In both of the above, it was assumed that stability is established by brain mechanisms in response to stimulus input. This view, however, was effectively challenged by James Gibson (1966), who attributed the stability of realworld percepts to the tuning of our visual system to unambiguous, invariant properties of stimulation that directly specify the source of the stimulation, the objects in the world and their spatial layout. From this perspective, there is no "blooming, buzzing confusion" and no isomorphism with respect to internal brain states. Percepts formed for natural stimuli are stable because they are uniquely specified by information in the stimulus, not because of intrinsic neural stabilizing mechanisms.

Although Gibson (1966) has had great impact on current thinking, bistable stimuli for which two qualitatively different patterns can be formed provide an important constraint on theories of perception. This is because it is very unlikely that stabilizing mechanisms have evolved just for the perception of bistable stimuli and are irrelevant to pattern formation for monostable stimuli. Our expectation is that understanding the perception of bistable stimuli will provide insights into pattern formation when perception is monostable.

\section{Motion Pattern Formation}

Solutions to the motion correspondence problem lie at the heart of pattern formation for global motion patterns. The motion correspondence problem is concerned with how pairwise correspondences are established across succcessive sets of simultaneously presented elements. The "problem" arises when different combinations of correspondence matches, and therefore, different motion paths are possible (Attneave, 1974; Kolers, 1972). A "solution," which entails establishing which of the alternative motion paths is perceived, is required for both monostable and bistable motion stimuli. For example, both horizontal and diagonal motions are possible for the translational motion stimulus illustrated in Figure 1A, but the solution to the motion correspondence problem always results in the perception of horizontal motion (Kolers, 1972); the translational stimulus is monostable. In contrast, both horizontal and vertical motions are possible for the motion quartet illustrated in Figure 1B, and there are two solutions to the motion correspondence problem-one resulting in the perception of horizontal motion, the other in the perception of vertical motion; the motion quartet stimulus is bistable.

The implications of bistability are examined in this study, using the motion quartet as an exemplary stimulus. As is illustrated in Figure 1B, the motion quartet is formed by simultaneously presenting two elements located in diagonally opposite corners of an imaginary rectangle, then simultaneously presenting two elements in the other diagonally opposite corners, then the first pair again, and so on (Chaudhuri \& Glaser, 1991; Hock, Kelso, \& Schöner, 1993; Hoeth, 1968; Kruse, Stadler, \& Wehner, 1986; Ramachandran \& Anstis, 1985; P. von Schiller, 1933). The elements are perceived to move either horizontally or vertically, but the two directions are never perceived at the same time, even though motion detectors selective for both horizontal and vertical motion are stimulated at the same time. Hock et al. (1993) varied the aspect ratio of the quartet, the vertical divided by the horizontal distance between element positions, and showed that (1) perception is bistable for aspect ratios not too different from 1.0either the horizontal or the vertical motion pattern is perceived for the same stimulus - and (2) perception spontaneously switches between the two patterns, the switching rate being highest for aspect ratios near 1.0.

\section{Adaptation and Switching}

In the past, perceptual switching for bistable stimuli has been attributed to the adaptation, or neural fatigue, of the perceived pattern and, at the same time, recovery from the effects of prior adaptation of previously perceived motions (Köhler \& Wallach, 1944; Spitz \& Lipman, 1962). On this basis, the pattern perceived through the course of these adaptation-induced changes is the one with the greater strength. This kind of account of perceptual switching does not require the introduction of a stabilization mechanism, but it is inconsistent with recent evidence concerning the influence of adaptation on the motion patterns perceived for the motion quartet. Hock, Schöner, and Hochstein (1996) have shown that both perceived and unperceived motion directions simultaneously adapt for the motion quartet; detectors responding selectively to motion in horizontal and vertical directions simultaneously adapt because they are simultaneously stimulated (hence, one is not recovering from prior adaptation while the other is adapting). ${ }^{1}$ In addition, Hock, Schöner, and Voss (1997) have determined that adaptation can increase the likeli- 
(A)

\section{Translational Motion Stimulus}

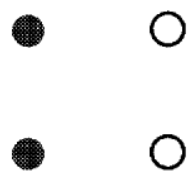

(B)

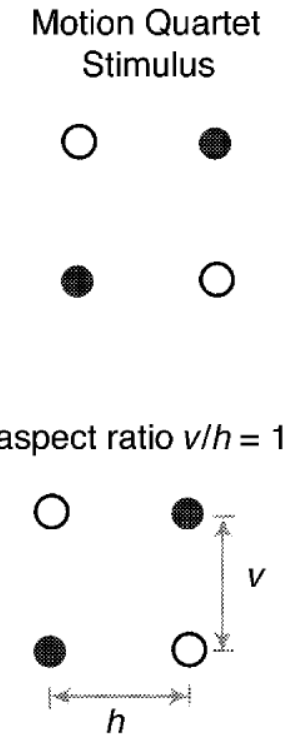

\author{
One Percept \\ is Possible
}
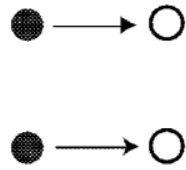

Two Percepts

are Possible

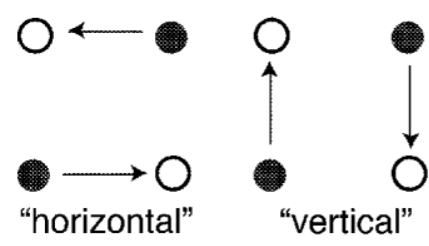

aspect ratio $v / h=2$

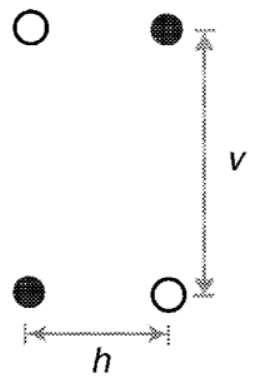

Figure 1. (A) Two frames of a monostable, translational apparent motion stimulus for which only one percept is possible. (B) Two frames of a bistable, motion quartet stimulus for which two percepts are possible.

hood of a switch for the motion quartet but that random fluctuations in activation are the agent for the switch. Hence, differential adaptation of the perceptual alternatives is not necessary for perceptual switching. ${ }^{2}$ It can be concluded from these studies that adaptation contributes to but does not provide a sufficient account of perceptual switching for bistable stimuli.

\section{Random Noise and Switching}

In signal detection theory (Green \& Swets, 1966), the presence of random noise is treated as a problem in the detection of monostable stimuli that produce activation levels of the same order of magnitude as the random fluctuations in activation in which they are embedded. Such approaches, however, do not address random influences on the perception of bistable stimuli, which typically entail stimulus-initiated activation levels that are much greater in magnitude than the noise. (At issue is which of two possible patterns is formed for a bistable stimulus, not the detectability of the patterns' attributes.) The signifi- cance of random influences on activation is particularly evident for the bistable motion quartet when stimulusinitiated activation is very similar for both horizontal and vertical motions (at an aspect ratio slightly greater than 1.0; Chaudhuri \& Glaser, 1991). Then, even very small random perturbations would change the relative activation of the two alternatives from one moment to the next.

At first glance, representing the activation of a detector (u) by the additive combination of a deterministic contribution from the stimulus $(S)$ and a stochastic contribution from random perturbations $(N)$ might seem sufficient to account for the bistability of pattern formation when the horizontal and the vertical motions of the motion quartet are equally stimulated. However, it can be seen in Figure 2A that if this were the case, switches based on whether horizontal or vertical is more activated would be too rapid to account for the persistence of horizontal or vertical motion that characterizes perception during the extended intervals during which there are no switches. This would be the case even if there was mutual inhibition between the 

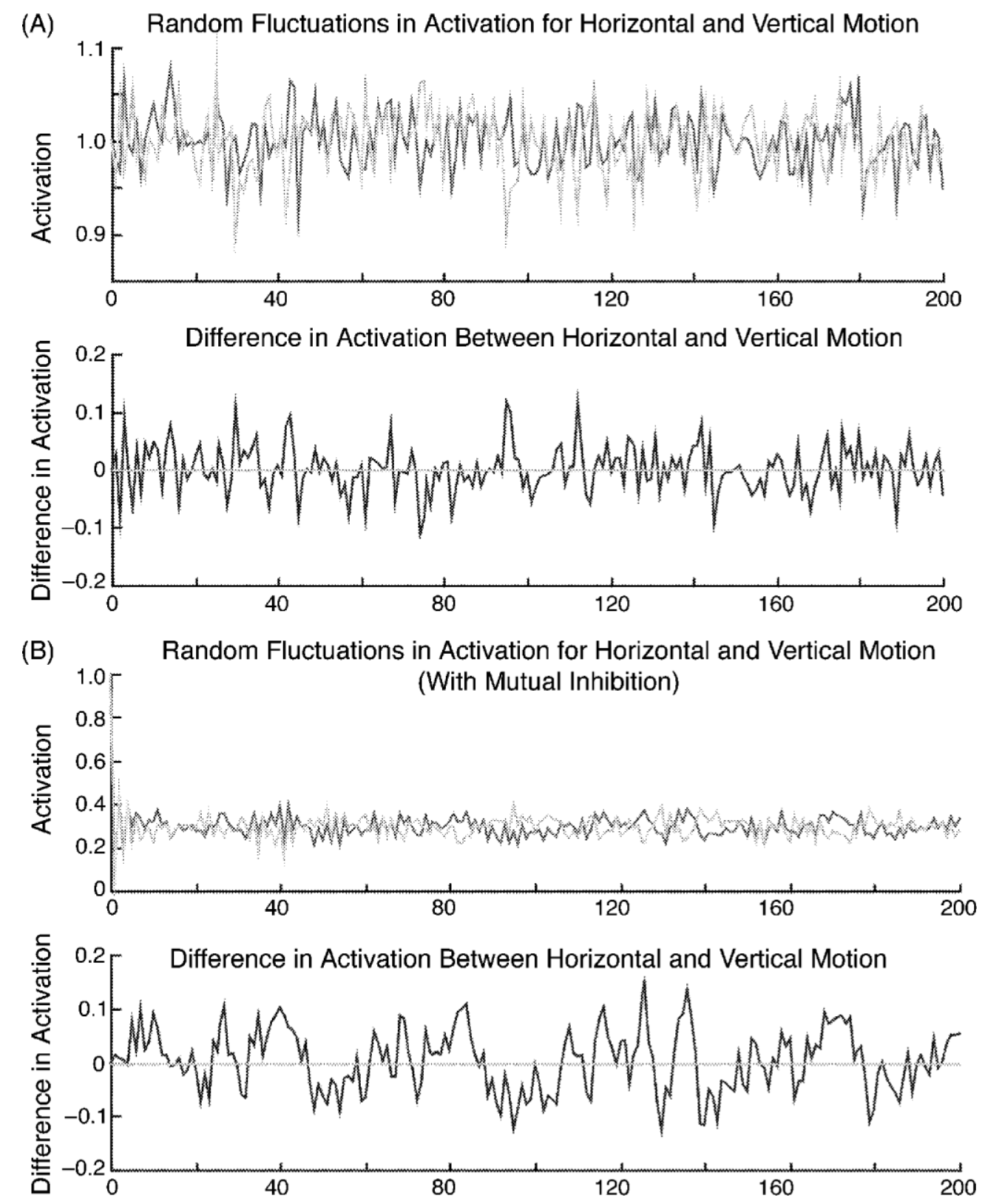

Figure 2. (A) Simulation of fluctuating activation for horizontal and vertical motion detectors based on the equation $u=S+N$, where both the stimulus activation, $S=1.0$, and the strength of the Gaussian white noise, $N=.03$, are the same for both motion directions. (B) Same as above, except that there is mutual inhibition between the two directions. The strength of the inhibitory coupling is 1.0.

detectors responding selectively to the horizontal and the vertical motion directions; activation then would be reduced for both directions, but their levels would not separate, and rapid noise-induced switching would remain (Figure 2B).

Random perturbations, therefore, can alter the relative activation of equally stimulated detectors, but they are not sufficient to produce persistent activational states unless perception is state dependent. That is, the formation of a temporally stable percept for a bistable stimulus requires that the activation state of a detector at each moment in time be predisposed by its immediately preceding activation state. The roles of interaction and stabilization mechanisms in establishing this state dependence is central to the dynamical theory developed in this article.

\section{Interaction and Stabilization}

There is abundant neurophysiologicalevidence consistent with the essential contribution of interaction to state dependence. The short- and long-range connectivity that is 
the basis for interactive detector networks has been neurophysiologically identified (Kisvárday, Tóth, Rausch, \& Eysel, 1997; LeVay, 1988; Ts'o, Gilbert, \& Wiesel, 1986), and Braitenberg (1978) has estimated that $95 \%$ of the input to each cortical neuron comes from connectivity with other cortical neurons. Neural connectivity also is indicated by the nonclassical behavior of receptive fields; that is, stimuli that lie outside the classical receptive field, and thus do not directly affect its activation, can modulate the activation produced by stimuli presented inside the classical receptive field (Allman, Miezin, \& McGuinness, 1985; Gilbert \& Wiesel, 1990). The idea of neural connectivity as the basis for the formation of stable patterns can be traced back to Hebb (1949) and has led to the development of a wide variety of interactive neural network models of pattern formation in motion perception (e.g., Chey et al., 1997; Dawson, 1991; Grossberg \& Mingolla, 1985; Grossberg \& Rudd, 1989; Nowlan \& Sejnowski, 1995; Wilson, Ferrera, \& Yo, 1992; Wilson \& Kim, 1994).

Because of this interconnectedness, each detector in a network is subject to the time-varying activational effect of the stimulus, activation-dependent interactive influences from other detectors, activation-dependentadaptation effects, and random perturbations that increase and decrease its activation over time. All these contributions to a detector's activation changes its interactive influence on other detectors, which in turn changes their influence on the detector's activation. This ongoing recycling of change, or recurrence, reflects the state dependence of the network; the evolving activation state of a detector depends on its previous activation state, as well as on the activation state of the detectors with which it interacts. If unchecked, recurrence can lead to either exploding levels of activation or no activation at all. However, when detectors have intrinsic mechanisms that stabilize their activation, recurrence results in the formation of stable percepts. On the basis of such stabilizing mechanisms, the distribution of activation over a population of interconnected motion detectors changes until the rate of change for each detector approaches zero (i.e., its activation stabilizes). It is only then that activation remains relatively unchanged for a sufficiently long period of time for a temporally persistent pattern to be realized in perception (Schöner \& Hock, 1995). In this way, the stimulus does not simply specify a particular activation level. Instead, it initiates a recurrent cycle of activation change that culminates with activation's settling into a stable distribution over ensembles of stimulated detectors. ${ }^{3}$

\section{CONCEPTUAL FOUNDATIONS OF A DYNAMICAL MODEL}

It is not the objective of this article to explore biophysical mechanisms responsible for the self-stabilization intrinsic to the operation of individual detectors (see Koch, 1999; Koch \& Poggio, 1987; Wilson, 1998). It suffices to characterize self-stabilization by an inverse functional re-
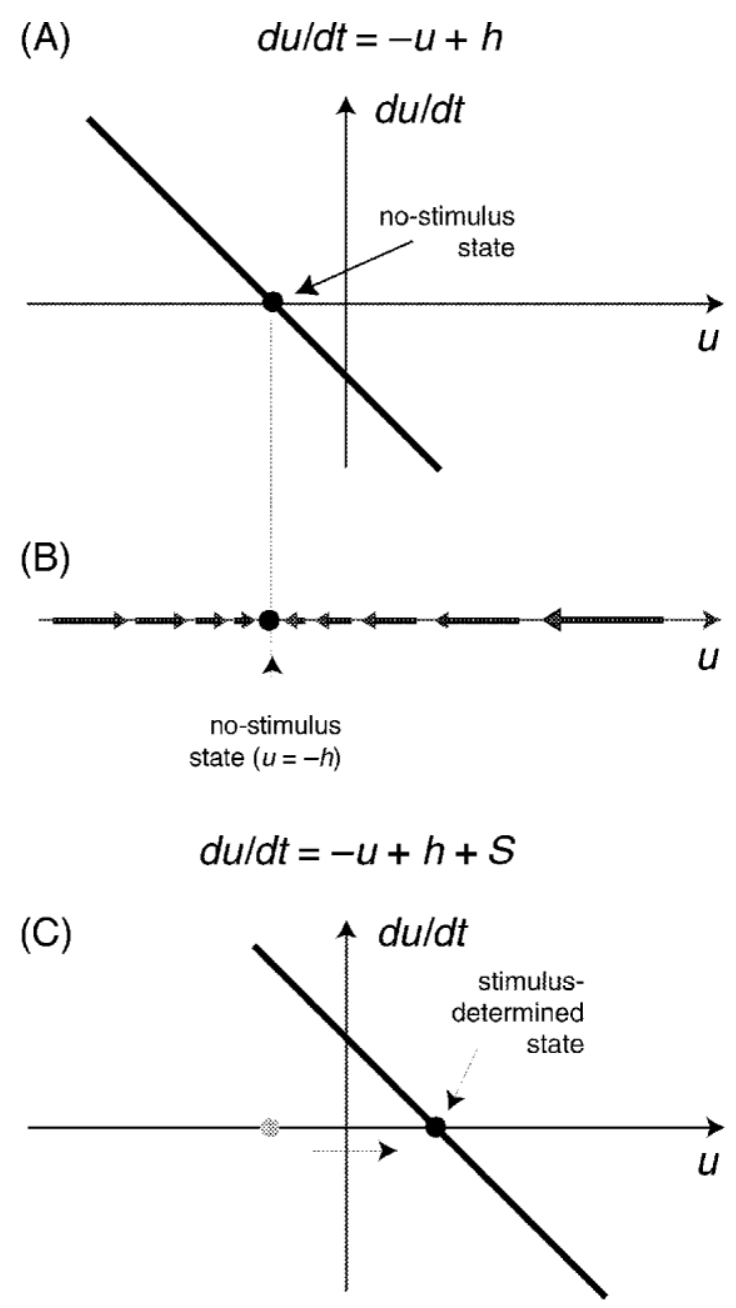

(D)

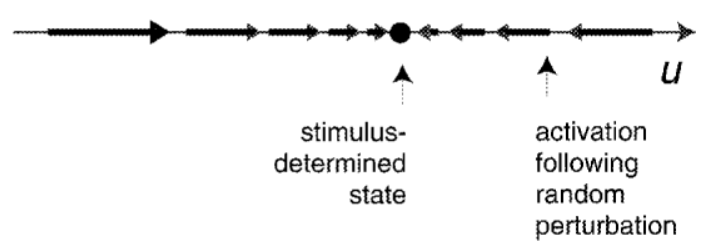

Figure 3. Illustration of how a detector behaves in the presence of a mechanism that stabilizes its activation. The rate of change of activation, $d u / d t$ (i.e., how activation is likely to change in the immediate future), depends inversely on the current state of activation $(u)$. This is shown (A) without stimulus-initiated activation and $(C)$ with stimulus-initiated activation. The rate of change in activation is negative (activation tends to decrease) if activation has been pushed upward by a random perturbation, and vice versa if it has been pushed downward. The one-dimensional vector field describes how activation, from whatever level it has reached because of a random perturbation, is likely to change in the immediate future (B) without stimulus-initiated activation and (D) with stimulus-initiated activation. Activation is brought toward the stable fixed point at a rate indicated by the length of vector. 


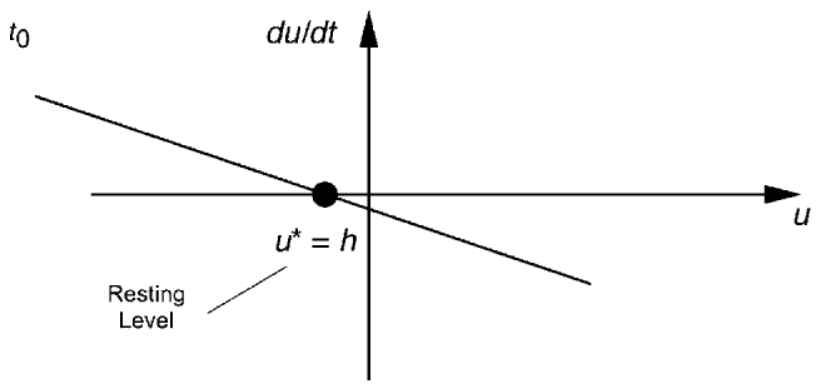

Fixed point shifts; activation increases from $u_{0}$ to $u_{1}$

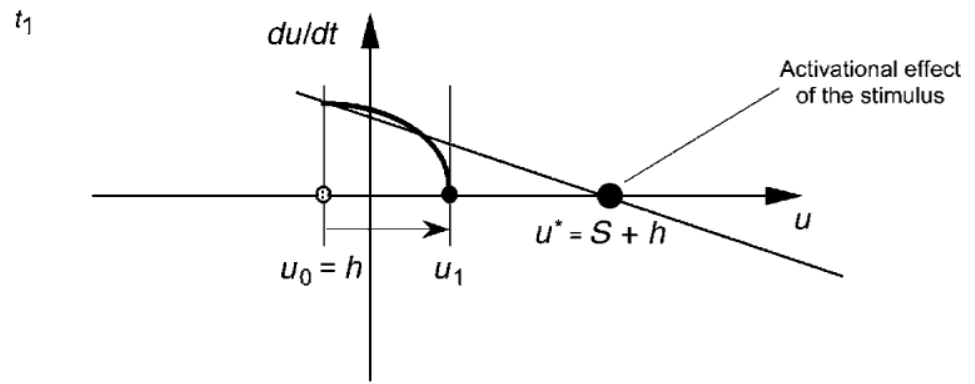

Activation increases from $u_{1}$ to $u_{2}$

$t_{2}$

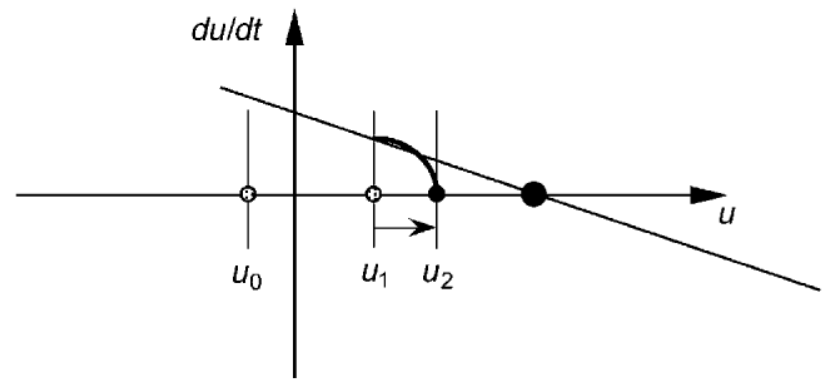

Activation increases from $u_{2}$ to $u_{3}$

$t_{3}$

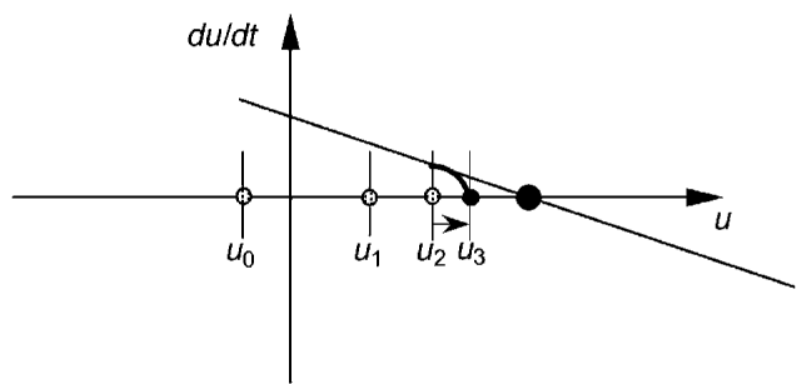

Figure 4. Illustration of how the stabilizing mechanism advances the activation of a detector from the no-stimulus resting level toward the fixed point level initiated by the presentation of a stimulus to which the detector is responsive. At successive moments in time, the increment in activation to a higher level $\left(u_{t+1}\right)$ is determined by the positive rate of change $(d u / d t)$ at the current activation level $\left(u_{t}\right)$. Activation approaches the fixed point exponentially, at a rate determined by the time scale (tau) of the stabilizing mechanism.

lationship between the detector's activation $(u)$ and its rate of change in activation $(d u / d t)$. As was indicated above, the "target" for the stabilizing mechanism is a zero rate of change in activation, not a particular activation level; the detector's activation is stabilized at the value of $u$ for which $d u / d t=0$ (where the function crosses the horizontal, "activation" axis in Figures 3A and 3C). In Figure 3A, there is no stimulus present to which the detector re- 


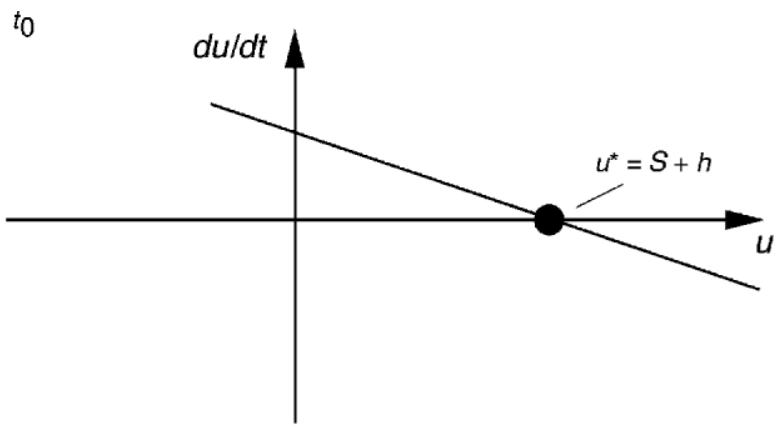

$t_{1}$
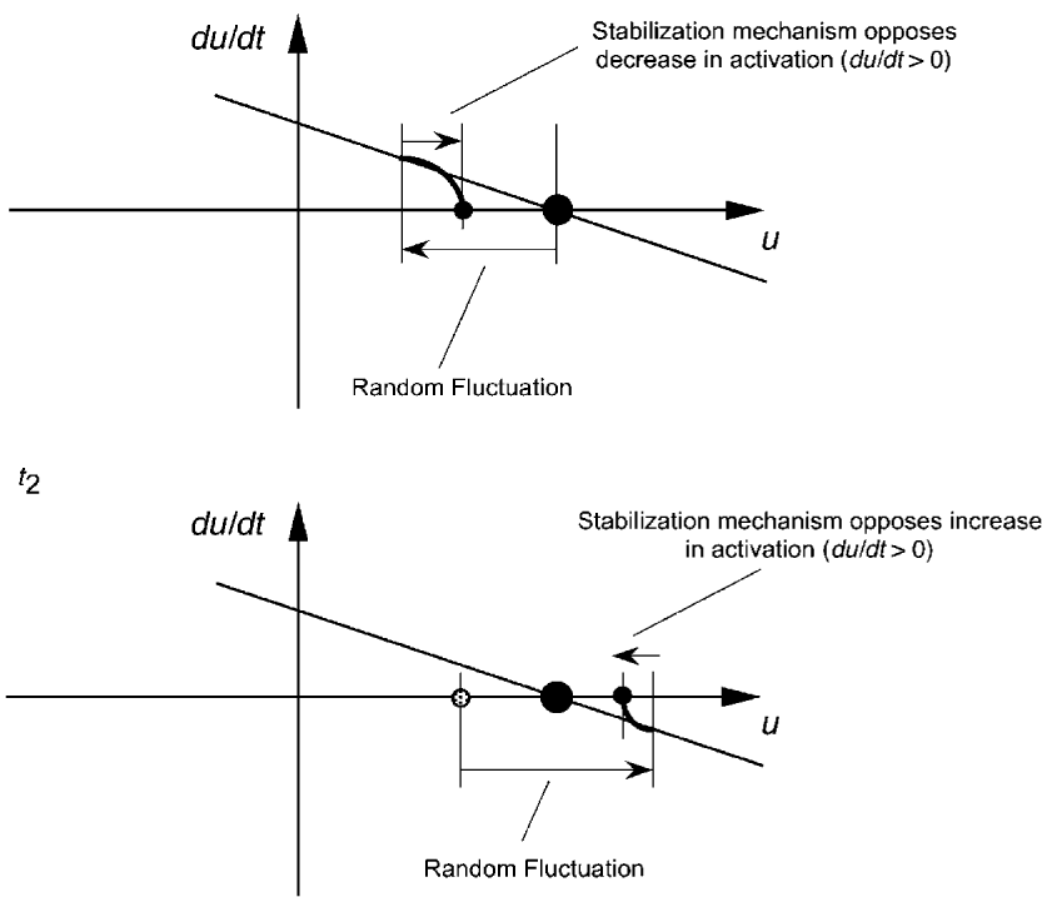

Figure 5. Illustration of how the stabilizing mechanism opposes perturbations that produce random fluctuations in activation that shift activation away from the stimulusdetermined fixed point. At successive moments in time, the activation value reached as a result of the perturbation is opposed by a change in activation determined by the rate of change $(d u / d t)$ at the perturbed activation value.

sponds, so activation is stabilized at the detector's resting level, $u^{*}=h$. The stable fixed point corresponding to this no-stimulus resting level is below the activation level of zero, which is arbitrarily designated as the threshold value required for perception.

When a stimulus is presented that is consistent with the directional selectivity of a motion detector, an activational change is imposed by the stimulus that shifts the stable fixed point to an activation value of $u^{*}=S+h$ (Figure 3C). As is illustrated in Figure 4, activation will increase at a rate determined by the time scale of the stabilizing mechanism, tau, until it reaches the stable fixed point value. If a random perturbation were to decrease activation relative to the fixed point activation level of the detector, the stabilizing mechanism would oppose this change by tending to increase activation in the immediate future. As is illustrated in Figure 5, successive random perturbations that increase and decrease activation would be opposed by the stabilizing mechanism, keeping activation close to the stable fixed point value determined by the stimulus.

\section{Vector Fields}

The dynamics for a local detector can be represented graphically by a one-dimensional vector field in which a vector is attached to each value of activation. Each vector points toward increased or decreased activation (in the immediate future), its magnitude indicating how strongly self-stabilizing mechanisms will shift activation in the indicated direction, either without (Figure 3B) or with (Figure 3D) stimulation. The vector fields illustrate that, start- 
(A)

\section{Symmetrical Stimulation}

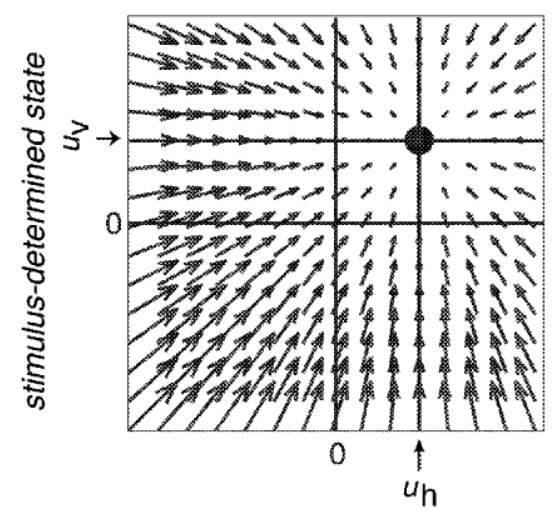

stimulus-determined state

(B)

\section{Asymmetrical Stimulation}

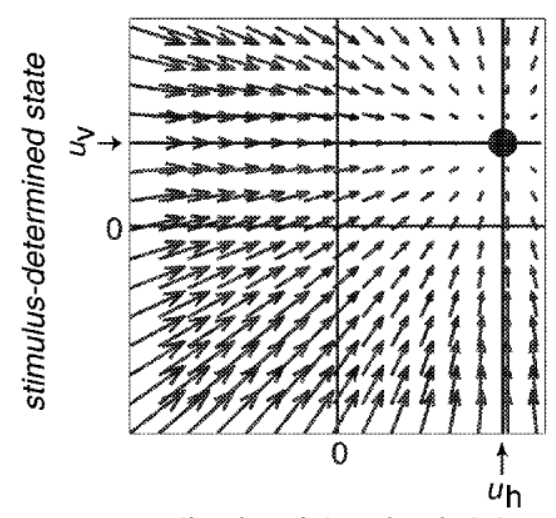

stimulus-determined state

Figure 6. Joint two-dimensional vector fields for the variables, $u_{\mathrm{v}}$ and $u_{\mathrm{h}}$, representing the activation of vertical and horizontal motion detectors. The vector field is formed by the vector addition of two one-dimensional vector fields (a one-dimensional vector field is illustrated in Figure 3D). The stable-fixed point for the activation variables is indicated by the large black dot. (A) Vector field when the activation of horizontal and vertical motions are equal. (B) Vector field when activation is asymmetrical (in this example, there is greater activation of horizontal than of vertical motion).

ing from any possible activation level resulting from a random perturbation, activation is likely to evolve in time (following the arrows) to the vicinity of the stable fixed point. The decreasing size of the arrows in the vector field diagrams reflects the exponential decrease in the size of the activation change produced in reaction to a perturbation as the stabilizing mechanism brings activation back toward the fixed point value. The rate of decrease depends on the value of tau.

\section{Global Motion Patterns}

The formation of a global motion pattern entails (1) the simultaneous stimulation of detectors at different spatial locations (there is more than one local motion) and (2) the modification of activation levels for the stimulated detectors by mutual interaction. If the global pattern is composed of both horizontal and vertical motion components (as is the case for the motion quartet), activation variables $u_{\mathrm{h}}$ and $u_{\mathrm{v}}$ each can be described by a one-dimensional vector field with a stable fixed point. They also can be jointly represented by a two-dimensional vector field with a single stable fixed point (with equal stimulus-initiated activation for $u_{\mathrm{h}}$ and $u_{\mathrm{v}}$ in Figure 6A; with greater activation for $u_{\mathrm{h}}$ than for $u_{\mathrm{v}}$ in Figure 6B). Each vector in the joint field is determined by the vector sum of each detector's stabilization-determined opposition to perturbations that shift activation away from its fixed point values.

Vector fields representing interactions among horizontal and vertical detectors are based on the assumption that the contributions of a local detector to the activation of other local detectors depends monotonically but nonlinearly on its activation. At detector activation levels near zero, the greater the activation of a detector, the greater its interactive influence on other detectors (i.e., interaction increases with activation). However, at more negative and more positive activation levels, changes in the activation of a detector have relatively little effect on the magnitude of its interaction with other detectors. This form of nonlinearity, which is adequately represented by a sigmoidal function (Grossberg, 1973), is illustrated in Figure 10.

Vector fields representing the effects of inhibiting interactions among local motion detectors are presented in Figure 7. For the motion quartet, an increase in the activation of horizontal motion detectors $\left(u_{\mathrm{h}}\right)$ means that the activation of vertical motion detectors $\left(u_{\mathrm{v}}\right)$ will decrease in the immediate future. The negative change in activation for vertical motion is indicated by the downward arrows in Figure 7A. (The magnitude of the change, as indicated by the length of each arrow, is determined by the previously described sigmoidal nonlinearity; the inhibitory influence of the horizontal detector is large when it is positively activated, small when its is negatively activated, and intermediate for activation values near zero.) The complementary inhibiting effect of vertical activation on activation for horizontal motion is shown in Figure 7B, and the joint vector field describing their mutual inhibition is shown in Figure $7 \mathrm{C}$ (vector sums are taken at each point in the field).

One can anticipate from the divergent vector field in Figure $7 \mathrm{C}$ how nonlinear inhibiting interactions among the stimulated detectors can lead to two separate activation states (bistability), rather than to a single, monostable state. That is, mutual inhibitory interaction will tend to move activation toward either the upper left (high values of $u_{\mathrm{v}}$ and low, potentially subthreshold values of $u_{\mathrm{h}}$ ) or toward the lower right (high values of $u_{\mathrm{h}}$ and low, potentially subthreshold values of $u_{\mathrm{v}}$ ).

Depicted in Figure 8A is a joint vector field combining the vector field representing the symmetrical contribution of the stimulus (from Figure 6A) with the vector field representing mutual inhibitory interaction (from Figure 7C). The combined vector field has two stable fixed points, or attractors, reflecting the bistability of perception for the 
(A) Horizontal inhibits vertical

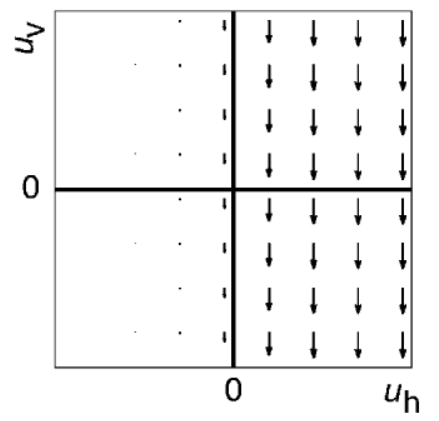

(B) Vertical inhibits horizontal

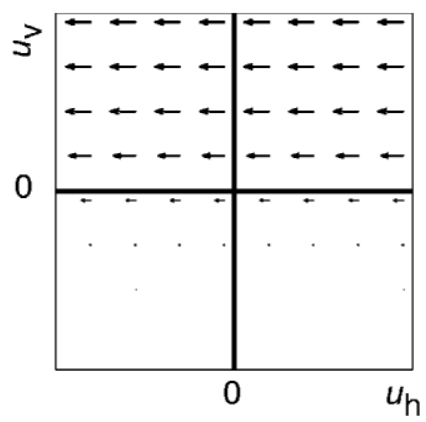

(C) Combined interaction

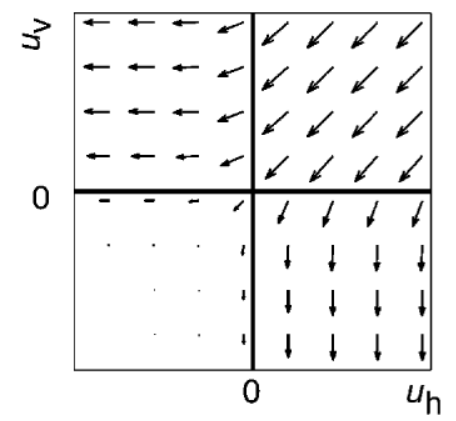

Figure 7. Joint two-dimensional vector fields representing inhibitory interactions among the activation variables. (A) The inhibitory influence of the activation variable for horizontal motion $\left(u_{h}\right)$ on the activation variable for vertical motion $\left(u_{\mathrm{v}}\right)$. (B) The inhibitory influence of the activation variable for vertical motion $\left(u_{\mathrm{v}}\right)$ on the activation variable for horizontal motion $\left(u_{\mathrm{h}}\right)$. (C) The combined vector fields, by vector addition, representing the mutual inhibitory interactions between the horizontal and the vertical activation variables.

motion quartet. Included in Figure 8A is a dark line that separates the vector field into two regions, the basins of attraction of the two stable fixed points. The attractorboundary (short for the boundary of the basins of attraction) emerges at those locations in the vector field from which either attractor can be reached following even a tiny random fluctuation in activation. The dynamics of the interacting detectors moves the activation state toward the stable fixed point within one or the other basin of attraction.

Changes in the aspect ratio of the motion quartet result in different levels of stimulation for horizontal and vertical motion. For example, when the horizontal interelement distance is shorter than the vertical interelement distance, there is greater stimulation of horizontal than of vertical motion detectors (Burt \& Sperling, 1981; Gilroy, Hock, \& Ploeger, 2001). This asymmetry is represented by the joint vector field for horizontal and vertical motion in Figure 6B. When combined with the vector field representing mutual inhibitory interaction (Figure 7C), the vector field representation illustrated in Figure 8B is formed. As compared with the symmetrical case, both stable fixed points are shifted toward higher horizontal and lower vertical activation, and significantly, the attractor boundary shifts away from the fixed point with greater horizontal activation and toward the fixed point with greater vertical activation. The shift of the attractor boundary is the result of a change in the locations in the vector field where the "forces" attracting activation to the alternative fixed points are balanced. As a result of these shifts of the fixed points and attractor boundary, one fixed point is closer to the attractor boundary, and the other is farther away. With sufficiently large asymmetries in stimulus-determined activation (for large or small aspect ratios of the motion quartet), one of the fixed points merges with the attractor boundary, resulting in the disappearance of one of the two basins of attraction. Perception is monostable beyond these critical values. Pattern formation, therefore, is probabilistic (subject to the effects of noise) only within the bistable range of the stimulus.

\section{Pattern Formation: What Is Perceived When a Stimulus Is Presented?}

The general answer to this question from a dynamical perspective is that the perceived motion pattern depends not just on the stimulus and interaction, but also on the immediately preceding activational state of the to-be- 
(A)

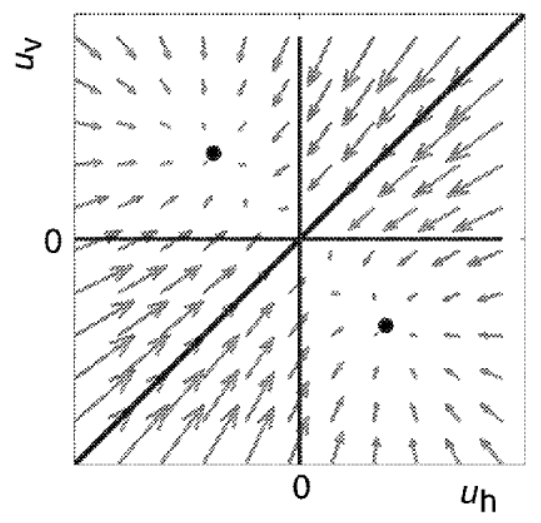

(B) Asymmetrical Stimulation

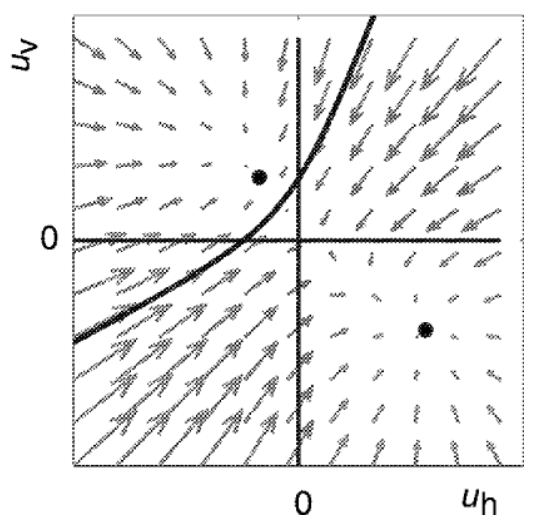

Figure 8. Joint two-dimensional vector fields that combine, by vector addition, the vector fields representing the stabilization of stimulus-initiated activation for the horizontal and the vertical variables (Figure 6) with the vector field representing the mutual inhibitory interactions between these variables (Figure 7C). The inhibitory field results in a bifurcation that leads to two stable fixed points: Horizontal activation is greater than vertical activation near one stable fixed point (lower-right quadrant of the vector field), and vice versa near the other (upper-left quadrant of the vector field). Threshold values for motion to be perceived are at an activation value of 0 , so in these examples, only horizontal motion is perceived when activation is near the stable fixed point in the lower-right quadrant, and only vertical motion is perceived when activation is near the stable fixed point in the upperleft quadrant. (A) When the combined vector field results from symmetrical stimulation of horizontal and vertical motion detectors, the stable fixed points are symmetrical with respect to $\mathbf{a 5}^{\circ}$ diagonal line through the center of the vector field. Horizontal and vertical motion are equally activated at their respective stable fixed points, and the $45^{\circ}$ diagonal also is the attractor boundary that separates the basins of attraction for the two stable fixed points. (B) When the combined vector field is based on asymmetrical stimulation of horizontal and vertical motion detectors, the stable fixed points are asymmetrical with respect to $4^{\circ} \mathrm{di}$ agonal line through the center of the vector field. In this example, horizontal motion is more strongly activated than vertical motion near their respective stable fixed points, and the attractor boundary is shifted toward the stable fixed point for vertical motion.

stimulated detectors. In the case in which there is no preceding stimulus, activation is stabilized at a value near the detectors' resting level and, therefore, below the threshold level required for perception. When a stimulus (e.g., a mo- tion quartet) is presented, both horizontal and vertical detectors are stimulated. As activation increases for these detectors toward stimulus-determined values, the inhibitory vector field depicted in Figure 7C is encountered. Activation is thereby "steered" by the inhibitory field toward one or the other of the two fixed points established by the combination of stimulation and interaction. The likely destination is determined by the location of the no-stimulus fixed point relative to the attractor boundary, as follows.

For asymmetric motion quartets with aspect ratios greater than 1.0, there is more stimulation of horizontal

(A)

What is perceived when a stimulus is presented?

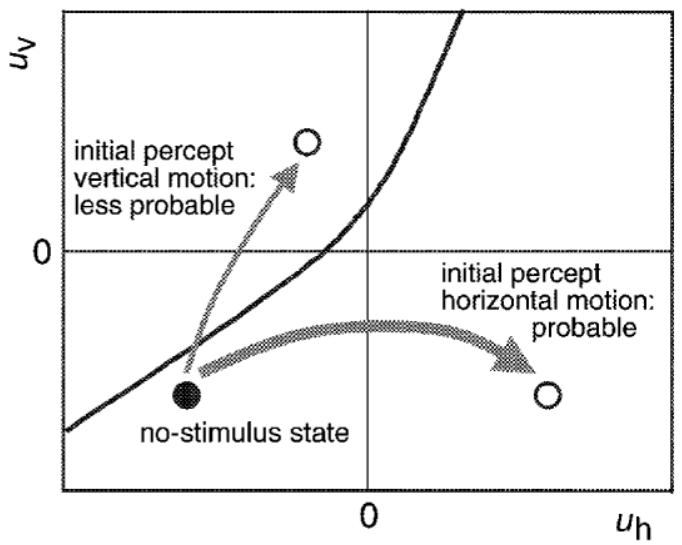

(B)

How stable is the initial percept?

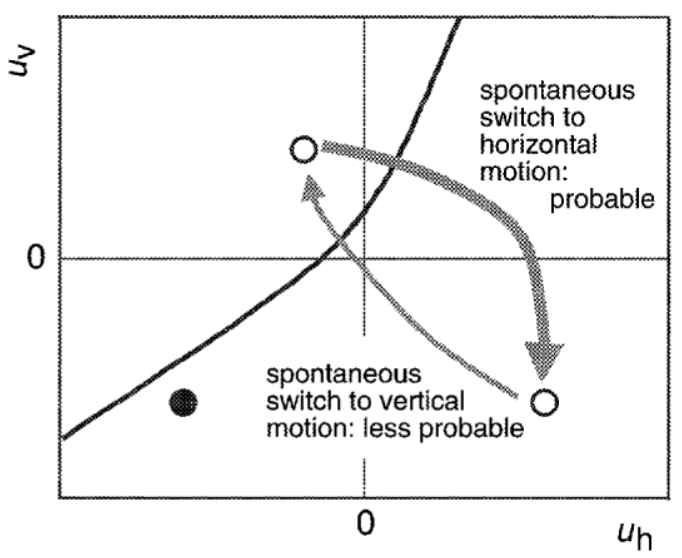

Figure 9. (A) When a stimulus is presented, the no-stimulus, stable fixed point (filled circle) is replaced by two stable fixed points (open circles) whose basins of attraction are separated by an attractor boundary (black line). What is perceived then depends on the position in the vector field of the no-stimulus fixed point relative to the attractor boundary. Percepts requiring random fluctuations for activation to cross the attractor boundary are less likely than percepts for which the stimulus-initiated fixed point is on the same side of the boundary as the no-stimulus fixed point. (B) Once a percept is established, its stability depends on the distance of the stable fixed point from the attractor boundary. This determines the size of the random fluctuation in activation that is necessary for activation to cross the attractor boundary and produce a spontaneous change in perception. Switches requiring large fluctuations are less likely than switches requiring small fluctuations. 
detectors than of vertical motion detectors, so the fixed points and attractor boundaries are shifted with respect to the no-stimulus resting level of activation (Figures $8 \mathrm{~B}$ and 9A). The no-stimulus fixed point lies on one side of the attractor boundary, in the basin of attraction for horizontal motion, so activation will move toward the fixed point for which there is above-threshold activation for horizontal motion and subthreshold activation for vertical motion. As is illustrated in Figure 10A, activation for horizontal motion detectors rises more quickly from the no-stimulus level than does activation for vertical motion detectors, because the former are more strongly stimulated. As a result, the activation of horizontal detectors reaches a level (still subthreshold for perception) where they begin inhibiting vertical detectors before the opposite occurs (Figure 10B). Although this usually leads to stabilization at the fixed point activation value for horizontal motion, it is possible, as the detectors' activation is increasing, for a random fluctuation of sufficient magnitude to suddenly change the detectors' relative activation, reversing the effect of the inhibitory vector field. As is illustrated by the vector field representation in Figure 9A, the fluctuation would shift activation across the attractor boundary, causing it to move to the other fixed point (resulting in the perception of the competing, vertical motion pattern).

For symmetric motion quartets (and in the absence of differential adaptation of horizontal and vertical motion), the no-stimulus fixed point lies on the attractor boundary. Activation values along the attractor boundary are very unstable, so even a very small random fluctuation is sufficient to move activation off the boundary (with a probability of .5 into one of the two basins of attraction). That is, activation for horizontal and vertical motion detectors rises at the same rate from the no-stimulus level, but random fluctuations occurring while activation is still subthreshold will result in one motion direction beginning to inhibit the other before the reverse occurs. In this way, each fixed point will be reached equally often, so either the hor-

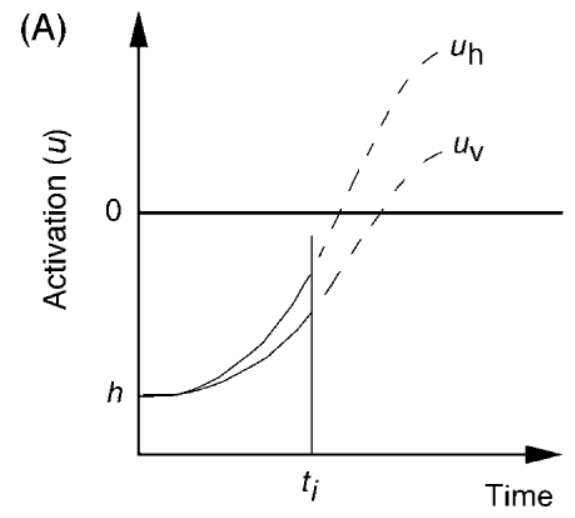

(B)

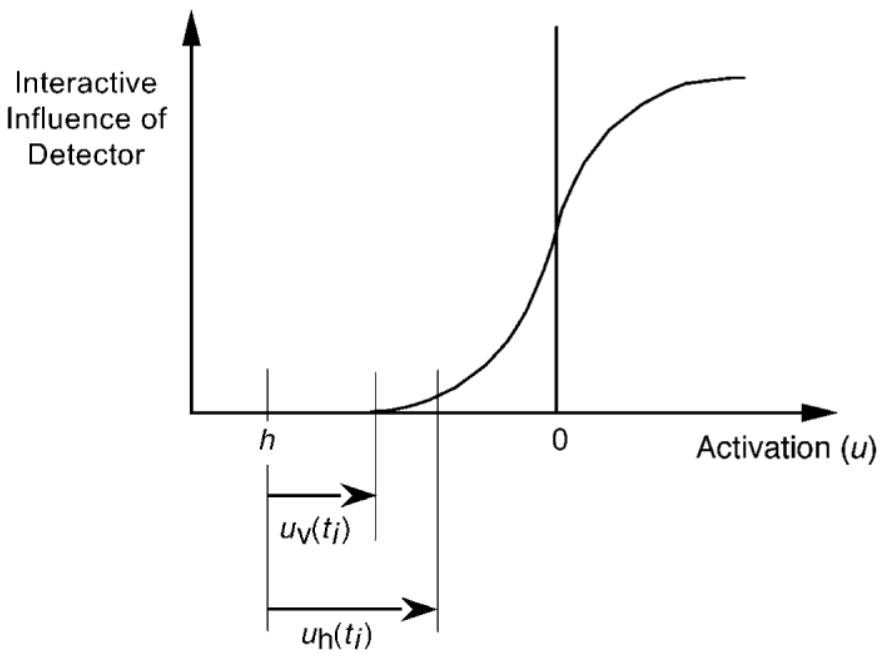

Figure 10. (A) The time course of activation change following the presentation of a stimulus. In this example, activation rises more quickly for $u_{\mathrm{h}}$ than for $u_{\mathrm{v}}$ because the horizontal motion detector is more strongly stimulated than the vertical motion detector. (B) As a result, $u_{\mathrm{h}}$ begins to have an interactive influence on $u_{v}$ before the reverse can occur. 
izontal or the vertical motion pattern will be formed with equal likelihood.

The dynamical solution to the motion correspondence problem for the motion quartet thus depends on which among the stimulated motion detectors is the first to initiate inhibition of its competitors as activation rises from the no-stimulus level toward one or the other of the two possible fixed point activation levels. The horizontal pattern is perceived when the activation of horizontal detectors is above and the activation of vertical detectors is below the threshold level required for perception (the lower-right quadrant in Figure 10A), and vice versa for the perception of the vertical motion pattern (the upper-left quadrant).

\section{Selective Adaptation and Dynamical Stability}

When a motion detector is activated by a stimulus, its fixed point activation level depends on the extent to which an attribute of the stimulus is consistent with its selectivity. Over time, adaptation results in a reduction of detector activation that is proportional to the level of activation initiated by the stimulus (Giaschi, Douglas, Marlin, \& Cynander, 1993), likely through the influence of activationsuppressing, inhibiting mechanisms. On this basis, adaptation provides a stimulus-dependentinhibitory contribution to the detector's activation that is specific to its selectivity. When the adapting stimulus is removed, activation relaxes back toward the detector's no-stimulus fixed point, but the adaptive effects of activation (its inhibitory vector field) will continue to be felt even as the activation producing it diminishes to levels that are subthreshold for the perception of the adapting stimulus. (The time scale of the adaptation is assumed to be substantially slower than the time scale of the detector's stabilizing mechanism, which governs how quickly the detector's activation changes.) The adaptation-induced inhibition shifts both the no-stimulus fixed point activation level and the attractor boundary initiated by the subsequently presented test stimulus. When the stimulation produced by the neutral test quartet is symmetrical, the fixed point that is reached and, thus, the pattern perceived for the test stimulus depend on the asymmetry of the adaptation, as follows.

There is much more adaptation of perceived than of unperceived motion directions when the adapting percept is highly stable (e.g., horizontal motion perceived when there is much stronger stimulation of horizontal than of vertical motion detectors). The highly asymmetrical inhibitory vector field resulting from the adaptation will substantially shift the attractor boundary established by the neutral test stimulus and the location of the no-stimulus fixed point relative to the attractor boundary (Figure 11A). As a result, the no-stimulus fixed point will fall well within the basin of attraction for vertical motion when the test stimulus is presented, so it is very likely that activation will move to the fixed point within that basin. The motion pattern perceived during adaptation would have a strong influence on subsequent pattern formation.

Adaptation is less asymmetrical when the adapting percept is less stable (e.g., horizontal motion perceived when there is similar stimulation of horizontal and vertical motion detectors), so the inhibitory vector field resulting from the adaptation of horizontal and vertical detectors will have a less distortive effect on the attractor boundary established by the neutral test stimulus and the location of the no-stimulus fixed point relative to the attractor boundary (Figure 11B). The no-stimulus fixed point again will fall in the basin of attraction for vertical motion when the test stimulus is presented, but it will be closer to the attractor boundary, allowing greater opportunity for a random fluctuation to shift activation back into the basin of attraction for horizontal motion. The motion pattern perceived during adaptation would affect subsequent pattern formation, but not as strongly as when stimulation is asymmetrical.

It can be concluded that adaptation is never restricted to the motions perceived during the adaptation phase of the experiment; it occurs for unperceived as well as perceived motion directions. What varies is the selectivity of the adaptation. That is, the effect of adaptation on subsequent pattern formation is more selective for the pattern perceived during adaptation when there is a greater difference in adaptation between perceived and unperceived motion directions. This depends on the stability of the adapting percept (Hock et al., 1996).

\section{Perceptual Continuity: Hysteresis}

Perceptual continuity depends on there being a smooth transition between the activation state established by the current stimulus and the new activation state that will be forthcoming as a result of a change in stimulation. A clear example is provided by the hysteresis observed for the motion quartet when its aspect ratio is gradually increased or decreased. When the aspect ratio favors the perception of the horizontal motion pattern, stabilizing mechanisms maintain that percept, resisting changes in aspect ratio that would switch perception to the vertical motion pattern. In terms of the vector field representation, changes in parameter do not require that the activational state of the stimulated detectors be reestablished following a return to nostimulus activation values. Instead, the activation state remaining from the preceding parameter value keeps activation within the same basin of attraction after the aspect ratio has changed (and the stable fixed point and attractor boundary of the dynamical representation are slightly shifted). This state dependence continues through a series of changes in aspect ratio, the percept remaining the same even as the values of aspect ratio are reached that would otherwise favor the competing percept (Hock et al., 1993).

The changes in activation and the dynamical representation initiated by successive changes in the aspect ratio of the motion quartet are illustrated in Figure 12. As the aspect ratio is gradually increased or gradually decreased, the stable fixed point of the initially established percept, as well as the current activation state, shifts toward lower levels of activation, the attractor boundary shifting in the opposite direction (toward the fixed point). An aspect ratio eventually is reached for which the fixed point 


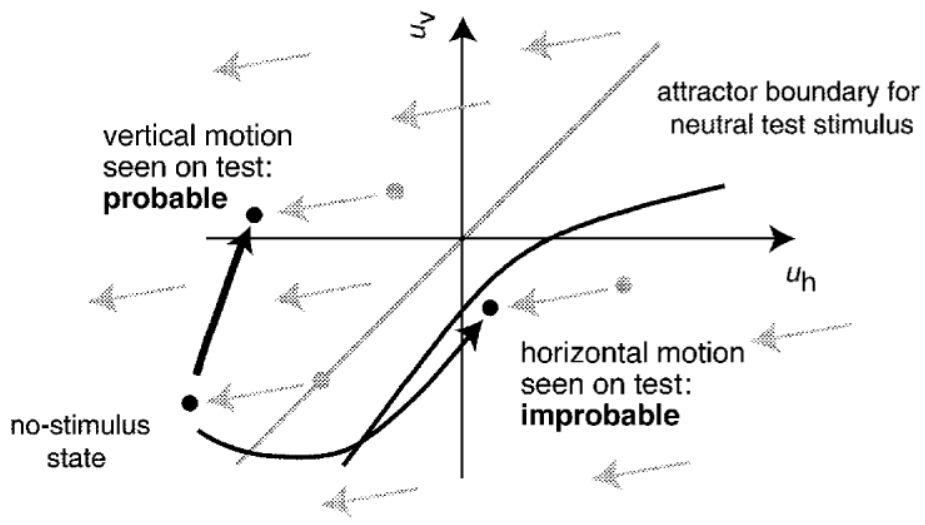

(B)

\section{Symmetrical Stimulation During Adaptation}

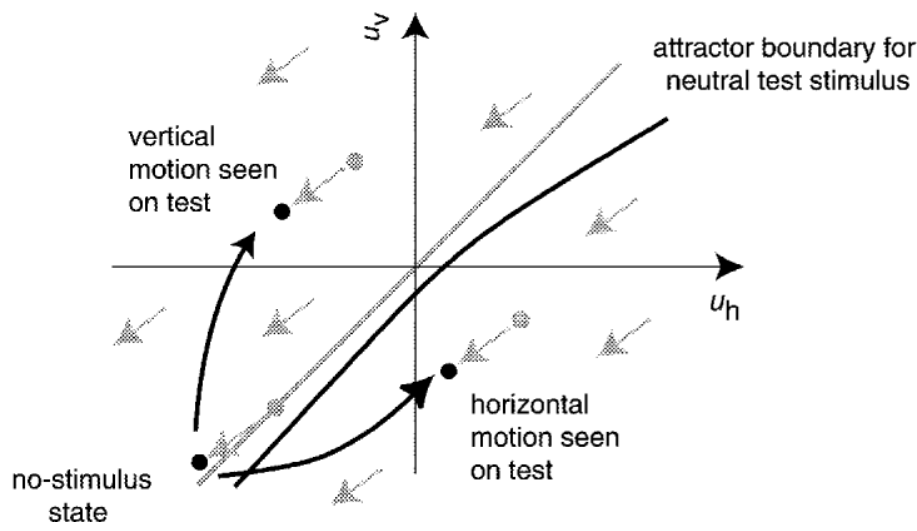

Figure 11. Illustration of how adaptation affects pattern formation for a bistable neutral test stimulus following an interval during which neither the adaptation nor the test stimulus is presented. The prior adaptation shifts the fixed points and attractor boundary of the vector field formed when the test stimulus is presented, as well as the no-stimulus activation level reached during the interval preceding the presentation of the test stimulus. (A) Asymmetrical stimulation (more horizontal than vertical stimulation) during the adaptation phase results in asymmetrical adaptation; the adapting vector field, indicated by light gray arrows, points mostly toward lower levels of horizontal activation (adaptation is strongly selective to the perceived horizontal motion). The no-stimulus activation state falls well within the basin of attraction for the vertical motion pattern, the perception of which is highly probable. (B) Symmetrical stimulation (equal horizontal and vertical stimulation) during the adaptation phase results in less asymmetrical adaptation than that shown in panel $\mathrm{A}$; the adapting vector field points toward lower levels of horizontal and vertical activation (adaptation is less selective to the perceived horizontal motion). The no-stimulus activation state falls near the attractor boundary, so given the occurrence of fluctuations large enough for activation to cross the attractor boundary, the perception of horizontal motion is more likely than that shown in panel $A$.

merges with the boundary, whereupon there is a bifurcation marked by a sudden switch to a much different activational state near the fixed point of the only remaining basin of attraction. Hysteresis occurs when two such bifurcation points exist, one encountered by gradually increasing a relevant parameter, the other by gradually de- creasing the parameter. However, switches due specifically to such bifurcations are difficult to observe in the presence of random noise. This is because, as the fixed point gets closer to the bifurcation point, the likelihood increases that a spontaneous fluctuation will "prematurely" cross activation into the opposing basin of attraction, before the ini- 
(A)

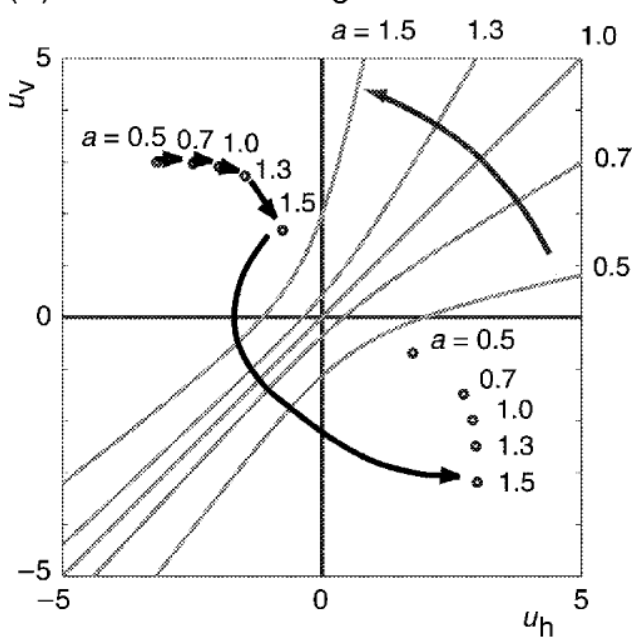

(B)

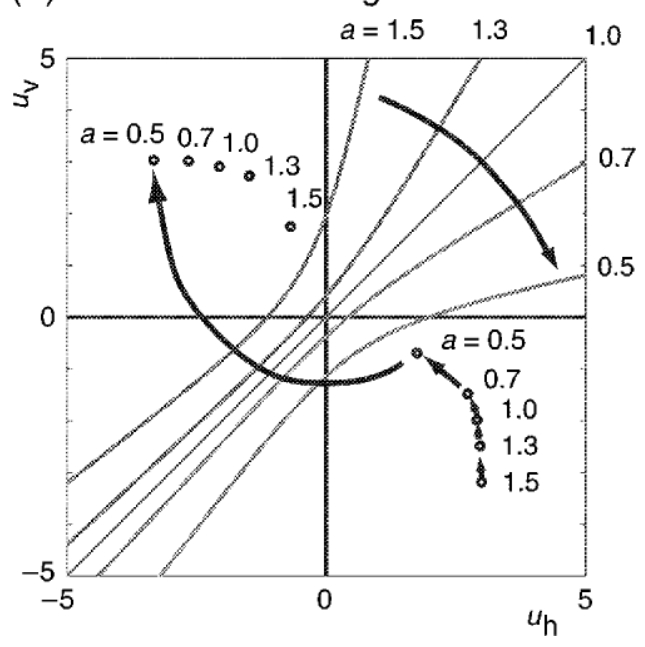

Figure 12. Hysteresis effect resulting from transitions between bistability and monostability. (A) As the aspect ratio is increased during ascending trials, the stable fixed point for vertical motion moves toward the attractor boundary, and the attractor boundary moves toward that stable fixed point. There is then a bifurcation. The fixed point disappears as it merges with the attractor boundary, and there is a sudden transition to the fixed point for horizontal motion, the only fixed point that remains (perception becomes monostable). (B) The same occurs during descending trials, except that the transition point is at a different aspect ratio. The two bifurcation points are reached when there are no random fluctuations that result in spontaneous jumps across the attractor boundary before it merges with a fixed point.

tially occupied basin of attraction has the opportunity to disappear (Hock et al., 1993).

\section{A DYNAM ICAL MODEL}

Accounting for phenomena such as hysteresis with the motion quartet provides a strong challenge to dynamical theory, because continuity must be established across temporal intervals during which relevant detectors are not stimulated. For example, rightward motion might be per- ceived at the top of the quartet when that motion is stimulated, but rightward motion will not be stimulated again at that location until after an interval of time during which leftward motion is stimulated instead. The dynamics must account for this periodic flow of activation between such alternately stimulated detectors, as well as for the switches between competing solutions to the motion correspondence problem that result from either random changes in activation or changes in stimulation.

Eight directionally selective motion detectors are required to represent the eight motions that can occur for the motion quartet over a succession of frame changes (Figure 13A). Four are stimulated during the first frame change, the other four, with the opposite directional selectivity, are stimulated during the second frame change, and so on, back and forth. The eight dynamical equations required to account for the time-varying activation of each motion direction are presented in the Appendix. Each of the eight activation variables is influenced by interaction with the

(A)

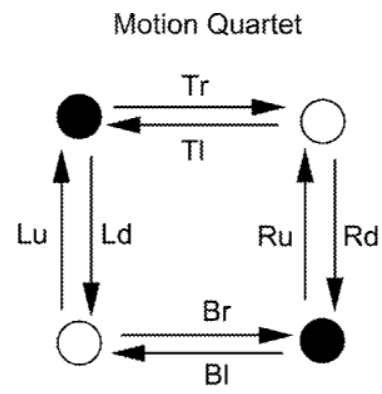

(B)

Translating Rod

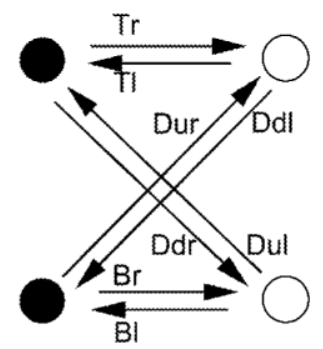

(C) Translating Rod With Illuminated Bars Along Diagonal Motion Paths

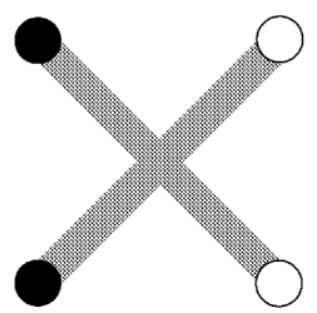

Figure 13. (A) Eight motion directions for the motion quartet stimulus, the basis of Simulations 1-8. (B) Eight motion directions for the translating rod stimulus, the basis for Simulation 10. (C) Luminance bars that increase the activation of diagonal motion detectors for the translating rod, the basis for Simulation 11. 
other seven. The latter include (1) inhibitory interactions among orthogonal motion directions at each corner of the quartet that are stimulated during the same frame change, (2) inhibitory interactions among orthogonal motion directions at each corner of the quartet that are stimulated during successive frame changes, (3) inhibitory interactions between opposite motion directions on opposite sides of the quartet, (4) inhibitory interactions between detectors with motion in opposite directions over the same path, and (5) excitatory interactions between the same directions on opposite sides of the quartet. ${ }^{4}$

\section{Pattern Formation}

The results of simulations based on this dynamical model of motion pattern formation are contrasted with predictions based on Ullman's (1979) minimal mapping solution to the motion correspondence problem. Ullman has argued that solutions to the correspondence problem depend on the stimulus-determined affinity of potentially corresponding elements, the distance between pairs of elements constituting a particularly strong determinant of affinity that leads to the nearest neighbor solution (e.g., Burt \& Sperling, 1981; Hock et al., 1993; Shechter, Hochstein, \& Hillman, 1988; Ullman, 1979). Minimal mapping additionally depends on the modification of stimulus-determinedelement affinities by local split competition (when an element presented during one time interval has possible matches with two or more elements presented during the next time interval) and local fusion competition (when two or more elements presented during one time interval have the same element as a possible match during the next time interval). This competition is captured in the dynamical model by inhibitory interaction among motion detectors that respond selectively to different motion directions.

Ullman (1979) further specified that the effectiveness of split and fusion competition depends on the presence of differences in affinity for the pairs of elements defining the alternative motion paths; there is no competition with-

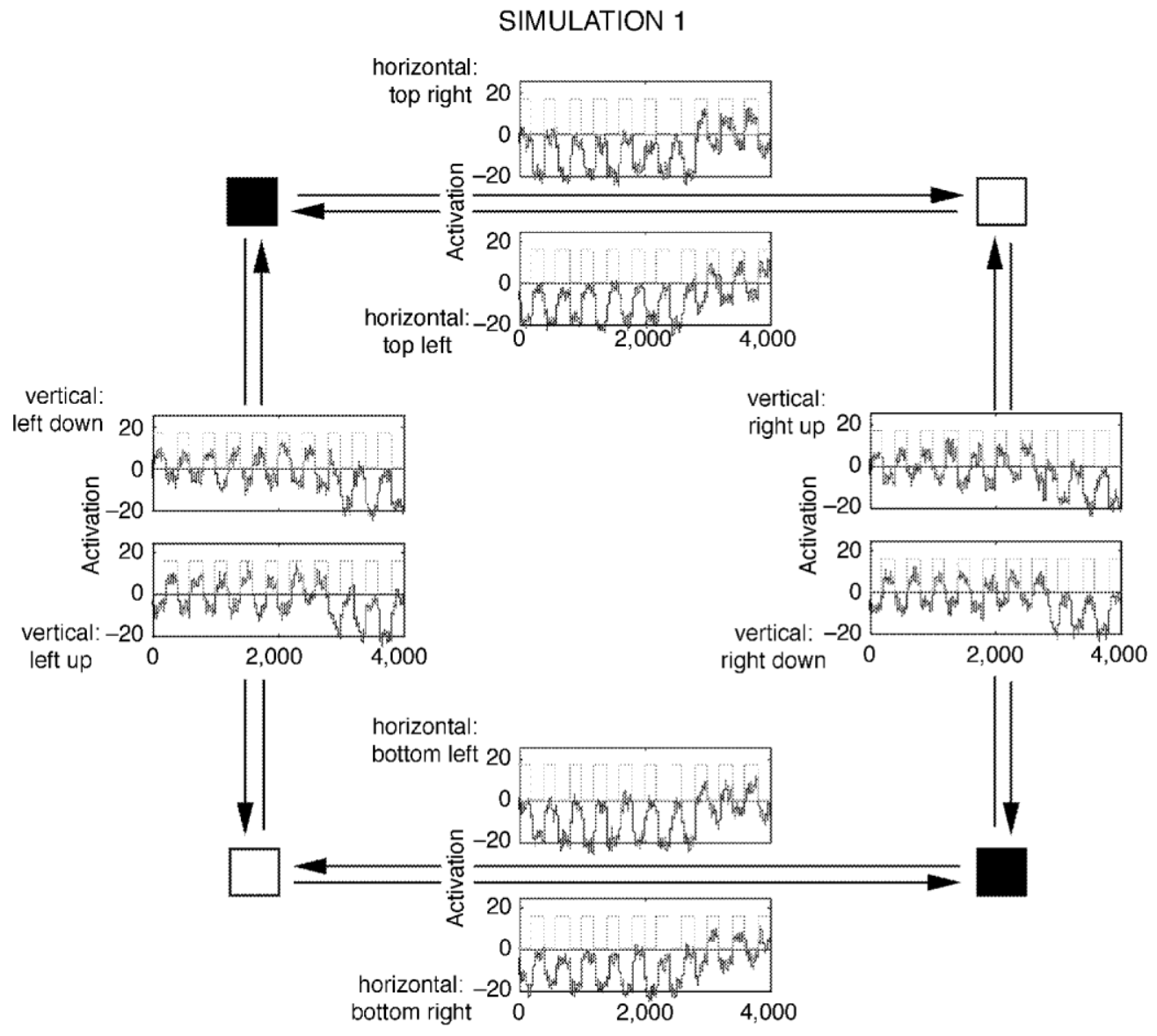

Figure 14. Simulation 1: the time-varying activation for each of the eight motion directions of the motion quartet. Either the horizontal or the vertical motion directions receive activation above the threshold level (zero) required for perception. The simulation is based on the equal stimulation of all motion directions $\left(S_{\mathrm{h}}=S_{\mathrm{v}}=16\right)$, the implementation of the detector interactions described in the text, and a level of noise strength $(N=0.7)$ sufficient to produce spontaneous changes between the horizontal and the vertical motion patterns. 
out differences in affinity, so the alternative motions of an element would be simultaneously perceived. With the additional constraint that all elements are covered (i.e., elements cannot simply appear and disappear), Ullman's minimal mapping solution determines the set of element pairings that minimizes cost (maximizes correspondence strength) over the entire set of possible element pairings. Dawson (1991) subsequently developed a connectionist model of motion correspondence that incorporates Ullman's principles, again stressing the relative distance between the elements (or equivalently, the relative velocity of the competing motions) as a primary constraint.

SIMULATION 2

(A)

Noise Strength $=0.07$

(equal horizontal/vertical stimulation)
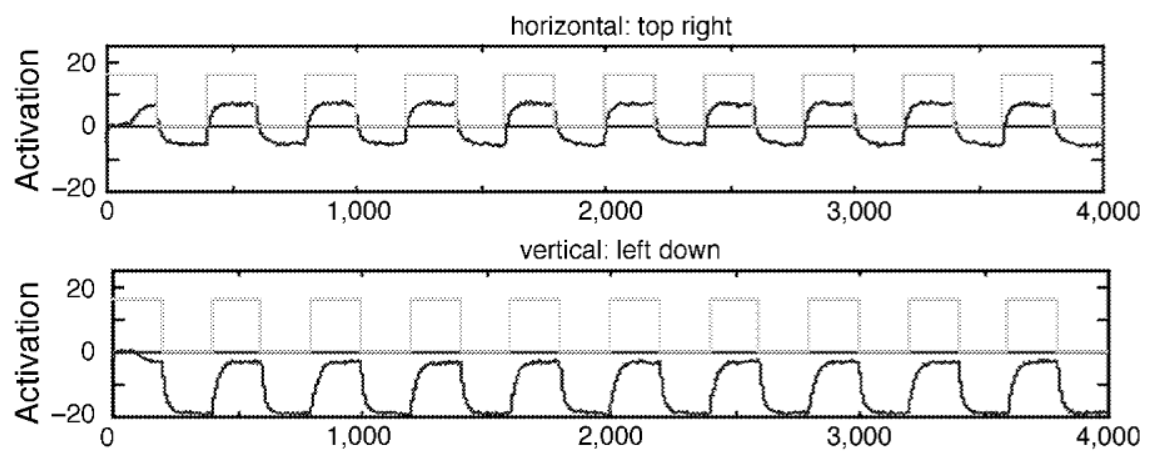

(B)

Noise Strength $=0$

(equal horizontal/vertical stimulation)

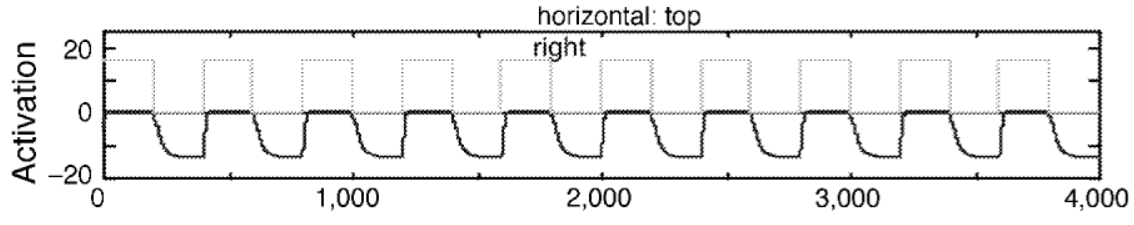

vertical: left down

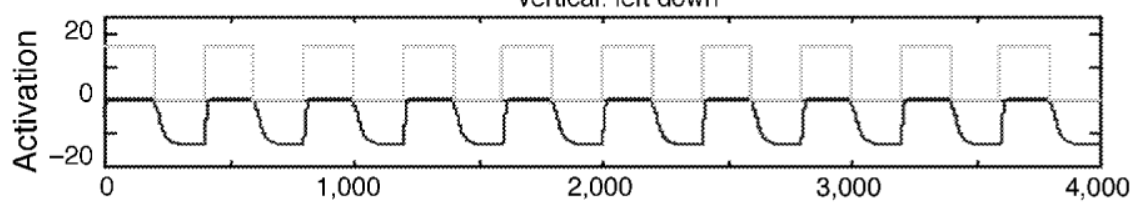

(C) (equal, but stronger horizontal/vertical stimulation)

horizontal: top right
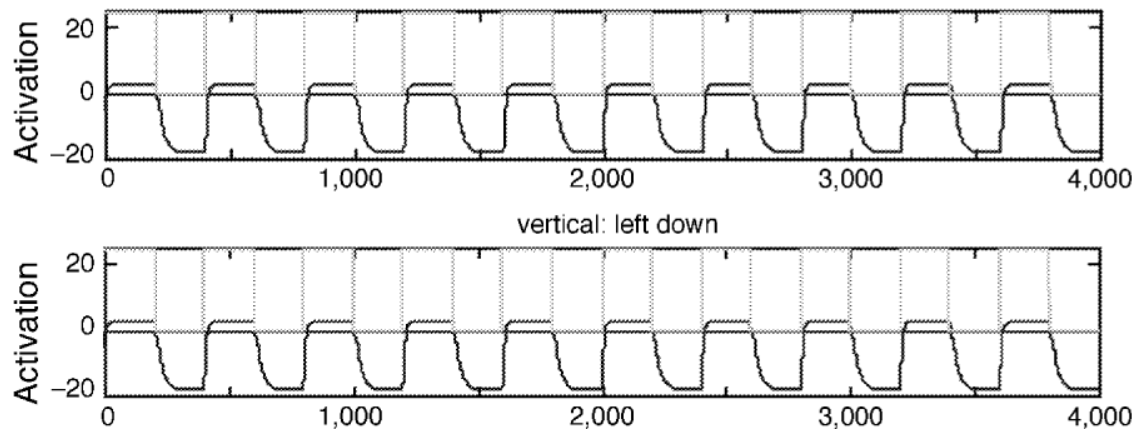

Figure 15. Simulation 2: the time-varying activation for two of the motion directions of the motion quartet (all eight are included in the simulation). Stimulation is equal for all eight motion directions, but there is less noise strength than in Simulation 1. (A) A reduced noise level $(N=\mathbf{0 . 0 7})$ is sufficient to result in the formation of either the horizontal or the vertical motion pattern. (B) Without noise $(N=0)$, there is always equal activation of all motion detectors. (C) This is the case even when stimulation of horizontal and vertical directions are greatly increased $\left(S_{\mathrm{h}}=S_{\mathrm{v}}=24\right)$. 
Simulation 1: Dynamical solution to the motion correspondence problem. The initial simulation in Figure 14 is based on the equal stimulation of all horizontal and vertical directions, the presence of interactions among the detectors (including those stimulated during the same and successive frame changes), and a level of noise sufficient to produce perceptual switching. It can be seen that for the first seven pairs of frame changes of the simulated trial, there is periodic flow of back-and-forth activation of vertical motion over the same path (alternating up/down motion as the two directions are alternately stimulated). For example, downward motion continues to be perceived on the right side of the quartet over the succession of frame changes during which it is stimulated, even though there are intervening frames during which upward motion is stimulated over the same motion path.

It also can be seen in Figure 14 that activation is closely coupled for the vertical detectors on the left and the right sides of the quartet (at an above-threshold level early in the simulated trial), as well as for all the horizontal detectors on the top and the bottom of the quartet (at a subthreshold level early in the simulated trial), signifying the perception of the vertical motion pattern. The simulation thus demonstrates how a perceived motion pattern can be embodied in the stable distribution of activation over a population of low-level, directionally selective motion detectors. That is, after each frame change, activation values stabilize near a fixed-point attractor, as described in the first part of this article. It is not necessary to evoke more specialized, higher order pattern detectors (e.g., detectors that respond selectively to shearing motions, which occur across opposite sides of the motion quartet; see, e.g., Tanaka, Fukada, \& Saito, 1989).

After the seventh pair of frame changes, there is a random fluctuation in the simulation of sufficient magnitude for the activation of all vertical detectors to drop below threshold and for the activation of all horizontal detectors to rise above threshold; that is, there is a spontaneous change from the perception of the vertical motion pattern to the perception of the horizontal motion pattern. Consistent with Hock, Schöner, and Voss's (1997) experimental results, this shows that the presence of random noise is sufficient to account for perceptual switching; differential adaptation of horizontal and vertical detectors is not necessary for switching, for the obvious reason that there was no adaptation, differential or otherwise, in this simulation.

Finally, the simulation indicates that inhibitory detector interactions, together with stabilizing mechanisms intrinsic to each detector, are sufficient to capitalize on noiseinduced differences in activation between the competing horizontal and vertical directions, separating their activation values (in contrast with Figure 2, for which there was no separation). Even though there was equal horizontal and vertical stimulation (in Ullman's [1979] terms, equal affinity), activation for either horizontal or vertical motion was above the threshold for perception, but never both simultaneously. This was consistent with experimental observation and contrary to the prediction of Ullman's minimal mapping theory that competing motions are perceived simultaneously when affinities are matched (because there is no split or fusion competition without differences in affinity).

Simulation 2: The effect of noise. In the preceding simulation, there was sufficient noise to produce solutions to the motion correspondence problem (either horizontal or vertical motion is perceived, never both at the same time) despite equal stimulation of horizontal and vertical motion detectors. Solutions also can be obtained with much less noise-for example, $10 \%$ of the noise strength in Simulation 1 (Figure 15A). However, in the complete absence of noise, there would be no solution to the motion correspondence problem (Figure 15B); when horizontal and vertical stimulation are matched, noise is necessary to break the symmetry. When both directions are more

\section{SIMULATION 3}

Noise-Induced Violation of Nearest Neighbor Principle horizontal: top right

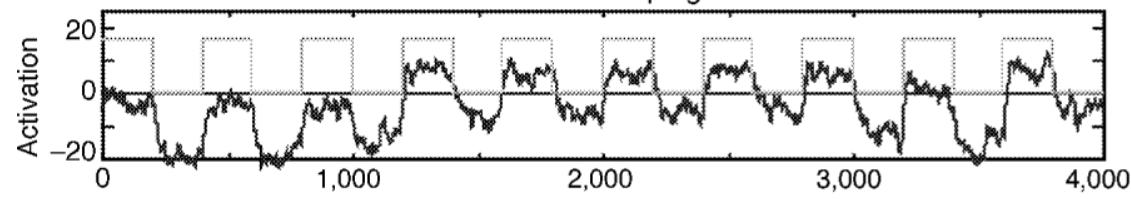

vertical: left down

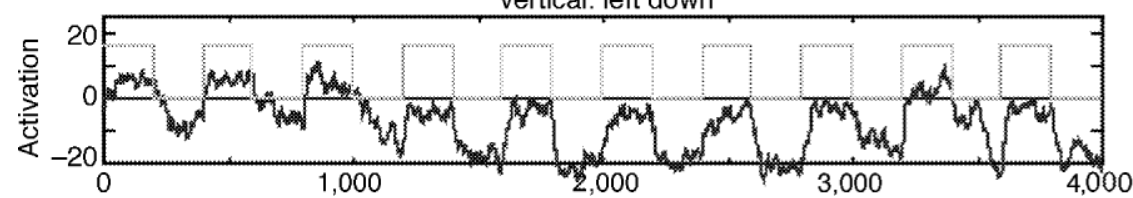

Figure 16. Simulation 3: the time-varying activation for two of the motion directions of the motion quartet when there is more stimulation of horizontal $\left(S_{\mathrm{h}}=16.5\right)$ than vertical $\left(S_{\mathrm{v}}=\right.$ 16.0) motion directions. Although the relative stimulus strengths favor the perception of horizontal motion, the presence of noise $(N=0.7)$ is sufficient to result in above-threshold activation for vertical motion, violating the nearest neighbor principle. 


\section{SIMULATION 4}

(A) Eliminating Interactions Among Simultaneously Stimulated Detectors (equal horizontal and vertical stimulation)

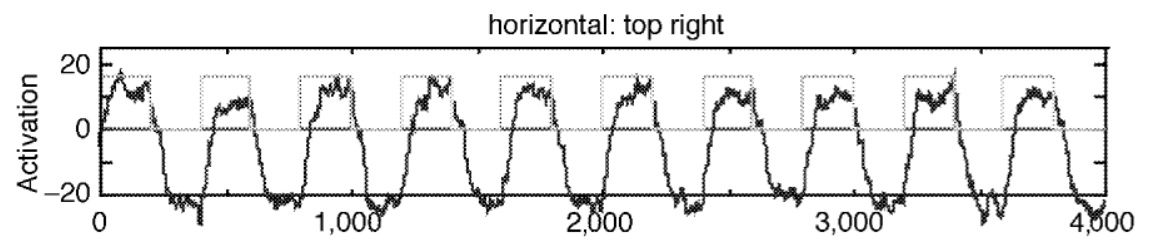

vertical: left down

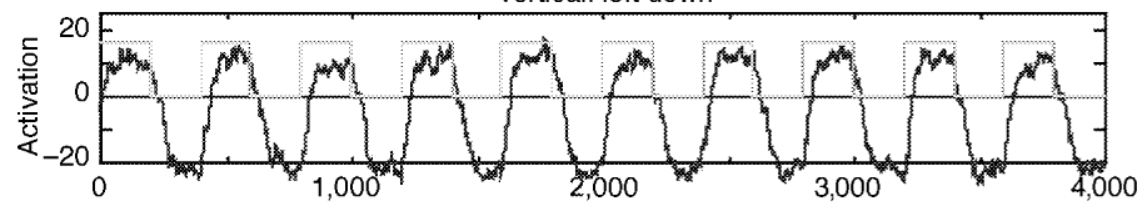

(B) Eliminating Interactions Among Simultaneously Stimulated Detectors (more horizontal than vertical stimulation)

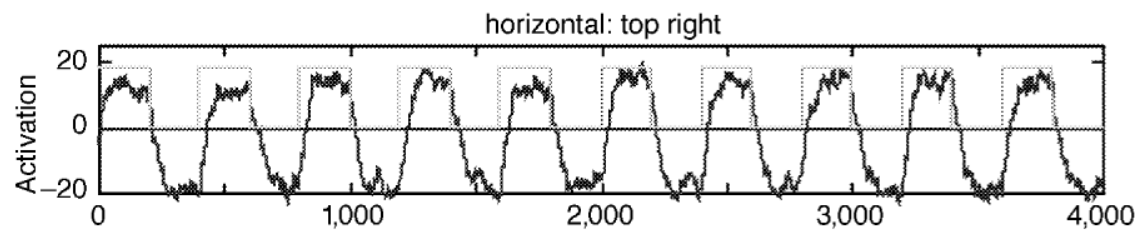

vertical: left down

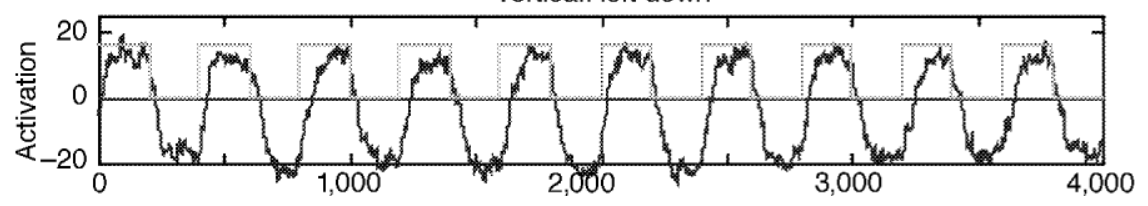

Figure 17. Simulation 4: the time-varying activation for two of the motion directions of the motion quartet when the interactive coupling of all simultaneously stimulated detectors is set to zero. Regardless of whether all motion directions are equally stimulated (A) or there is more stimulation of horizontal $\left(S_{\mathrm{h}}=18\right)$ than of vertical motion directions $\left(S_{v}=16\right)$, both horizontal and vertical detectors receive above-threshold activation.

strongly stimulated, equal above-threshold activation levels are obtained for both, incorrectly signifying their simultaneous perception (Figure 15C). Activation levels for this simulation are low because, in the absence of noise, neither motion direction is able to dominate, so the activation of both is greatly reduced by inhibition.

Neither Ullman's (1979) minimal mapping theory nor Dawson's (1991) connectionist version includes the random fluctuations that are necessary to account for solutions to the correspondence problem that occur in perception when stimulus-initiated activation is matched for competing motion directions. (In this set of simulations and those that follow, the graphs represent the time course of activation for only one horizontal and one vertical direction. The periodic flow of back-and-forth activation and the close coupling of all horizontal motions and all vertical motions reoccur in every simulation.)

Simulation 3: Noise-induced violations of the nearest neighbor principle. An important feature of the dynamical representation is that, contrary to minimal map- ping theory, it can account for the perception of motion patterns that violate the nearest neighbor principle (Hock et al., 1993). This is illustrated by Simulation 3 (Figure 16), which shows that the vertical motion pattern can be formed even though differences in motion path length result in more stimulation of horizontal than of vertical motion detectors. (Gilroy et al., 2001, have provided evidence for an inverse linear relationship between detector activation and motion path length.) Switching occurs relatively early in this simulation, reflecting the instability of patterns formed when the stimulus favors the competing pattern (see also the vector field representation in Figure 9B).

Simulation 4: The function of interactions among simultaneously stimulated detectors. Interactions among simultaneously stimulated detectors are the basis for solutions to the motion correspondence problem and, thus, the formation of motion patterns. It can be seen for Simulation 4 that removing detector interactions among simultaneously stimulated horizontal and vertical detectors results in their simultaneous above-threshold activation, 
SIMULATION 5

(A) Eliminating Interactions Among Successively Stimulated Detectors

(Noise Strength $=0.7$ )

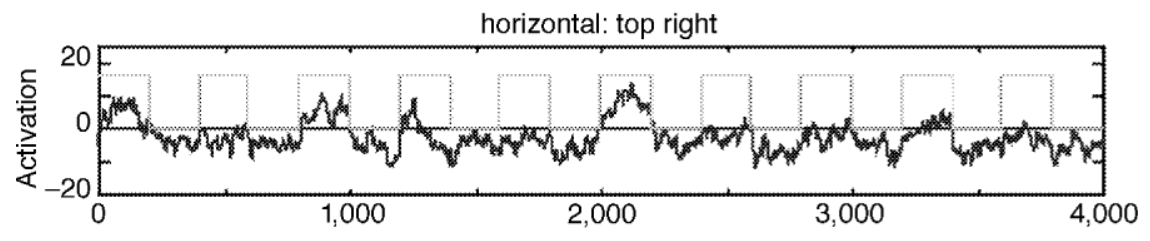

vertical: left down

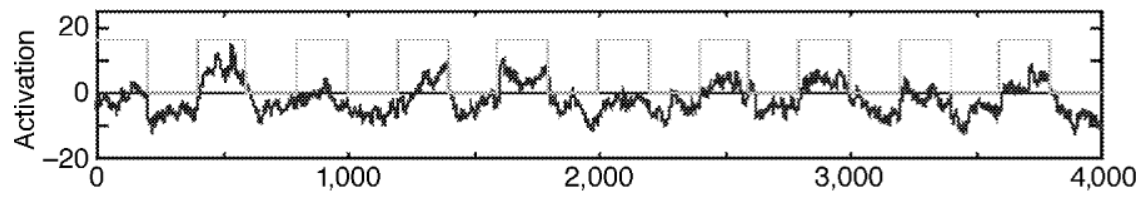

(B) Eliminating Interactions Among Successively Stimulated Detectors

(Noise Strength $=0.2$ )

horizontal: top right
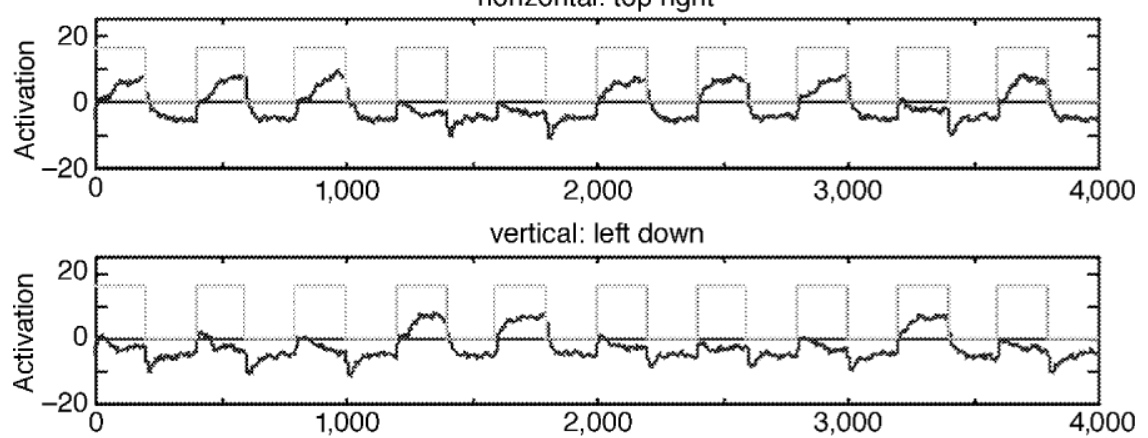

Figure 18. Simulation 5: the time-varying activation for two of the motion directions of the motion quartet when the interactive coupling of all sequentially stimulated detectors is set to zero. Horizontal motion and vertical motion are not perceived at the same time, but neither motion pattern is temporally persistent. This is shown for two levels of noise strength: (A) $N=0.7$ and (B) $N=0.2$.

regardless of whether horizontal and vertical motion are equally (Figure 17A) or unequally (Figure 17B) stimulated. This signifies the simultaneous perception of horizontal and vertical motion, a failure to solve the motion correspondence problem that never occurs for the motion quartet.

Simulation 5: The function of interactions among successively stimulated detectors. In contrast with the previous simulation, which removed interactions among simultaneously stimulated detectors, Simulation 5 removes interactions among motion detectors that are stimulated during successive frame changes. The motion correspondence problem continues to be solved following each frame change (the perception of either horizontal or vertical motion is signified), but there is no persistence of perception from one frame to the next (Figure 18A). That is, the pattern formed when the stimulus is presented (in this simulated trial, the horizontal motion pattern) immediately switches to the vertical motion pattern and then incoherently back and forth between the two. The same occurs when the simulation is done with less noise (Figure 18B).

The simulations thus demonstrate that "future shaping" interactions among successively stimulated detectors are the means by which pattern formation during a frame is influenced by the preceding activational state. Persistence does not occur without this state dependence.

\section{Continuity in Perception}

The preceding simulation shows that state dependence accounts for the continuity of a perceived motion direction even though there are intervening intervals during which that direction is not stimulated. The simulations that follow account for perceptual continuity despite changes in how strongly a motion direction is stimulated as compared with competing motion directions.

Simulation 6: Hysteresis. Hysteresis effects are simulated in Figure 19 by changing the aspect ratio of the motion quartet, gradually increasing or gradually decreasing the path length for vertical motion. This decreases or increases the stimulation of vertical detectors while the stimulation of horizontal detectors remains constant (the length of the horizontal motion path is fixed). The occurrence of hysteresis is indicated in the simulation by different switching points (denoted by arrows), depending on whether the initial aspect ratio favored the formation of 


\section{SIMULATION 6}

Hysteresis With Descending Trial

(decreasing vertical path length, increasing vertical activation)

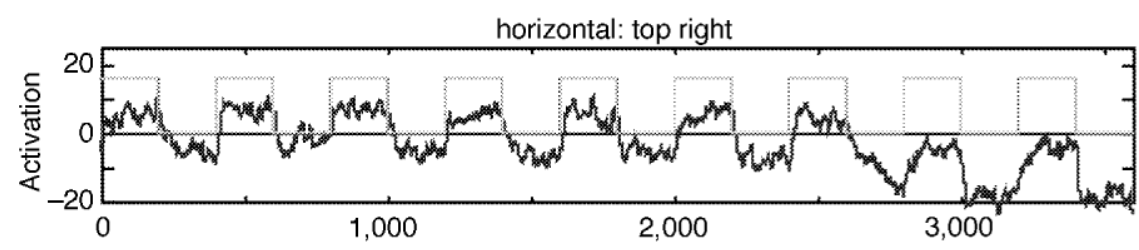

vertical: left down

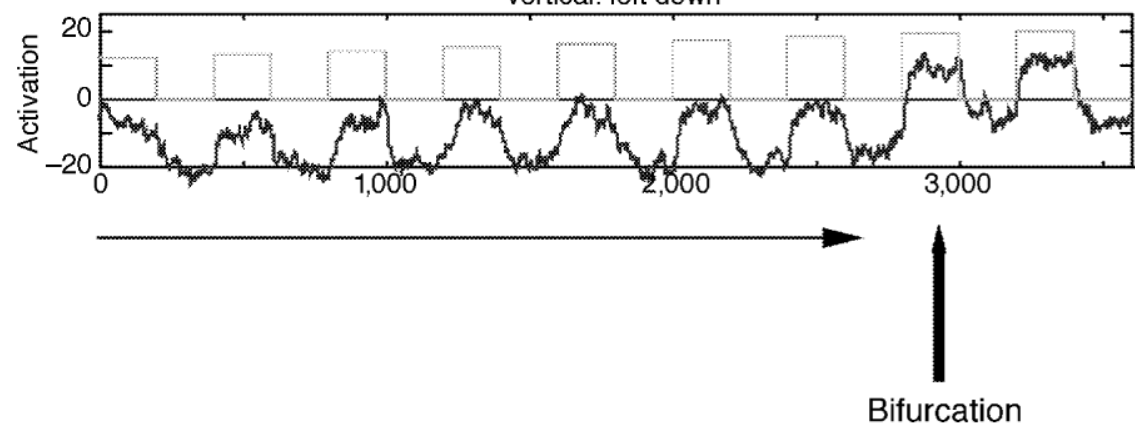

Hysteresis With Ascending Trial

(increasing vertical path length, decreasing vertical activation)
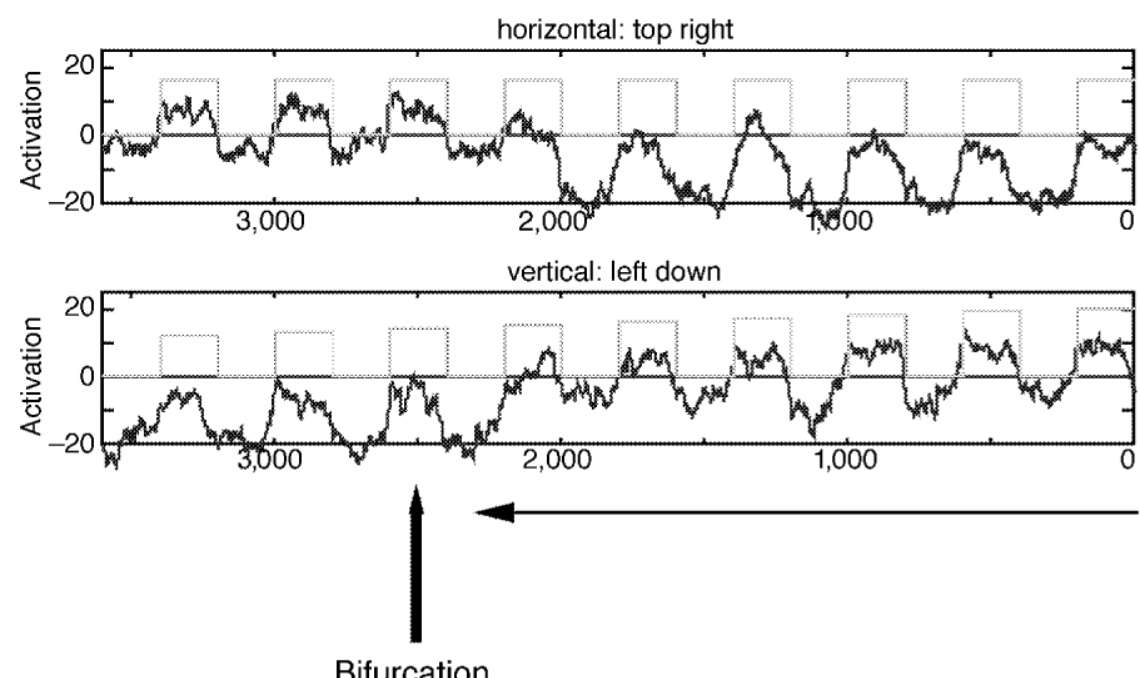

Figure 19. Simulation 6: the time-varying activation for two of the motion directions of the motion quartet when its aspect ratio is gradually decreased by decreasing the path length of vertical motion (A) or is gradually increased by increasing the path length of vertical motion (B). Hysteresis is indicated by switching points (bifurcations) occurring at different aspect ratios, depending on the direction in which the aspect ratio of the quartet is changed.

the horizontal or the vertical motion pattern (see also the vector field representation in Figure 12). For trials composed of a descending sequence of aspect ratios, the horizontal motion pattern is perceived despite decreases in the length of the vertical motion path relative to the length of the horizontal motion path to values favoring the vertical motion pattern, and vice versa for trials composed of an ascending sequence of aspect ratios (the frames with equal horizontal/vertical stimulation are in the center of each graph). The simulation is thus consistent with Hock et al.'s (1993) evidence for hysteresis. ${ }^{5}$ Perceived motion directions persist despite changes in path length that result in the greater stimulation of competing orthogonal motion directions and despite there being intervening frames during which the opposite motions are stimulated over the same motion path. Because the nearest neighbor con- 


\section{SIMULATION 7}

(A) Descending Trial Without Interactions Among Successively Stimulated Detectors (Noise Strength $=0.7$ )
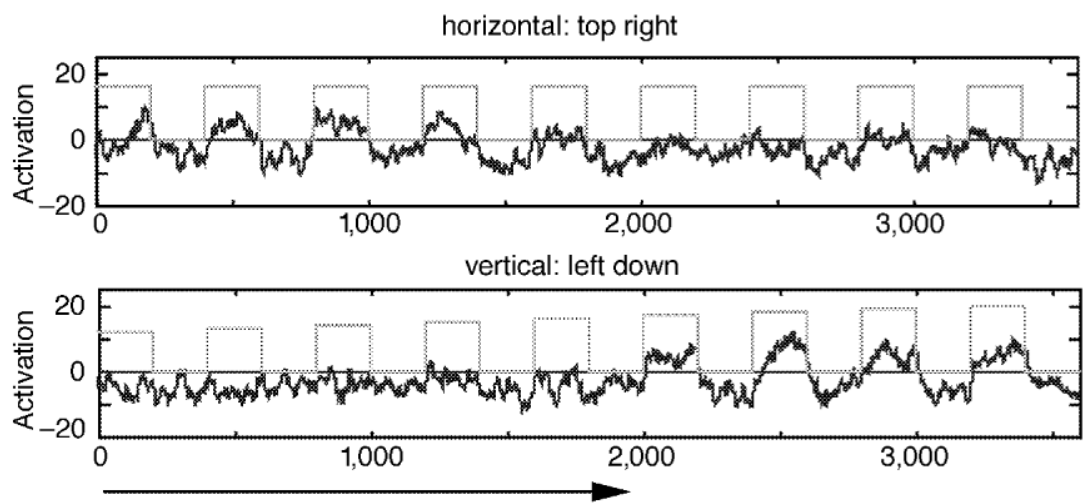

(B) Descending Trial Without Interactions Among Successively Stimulated Detectors (Noise Strength $=0.2$ )
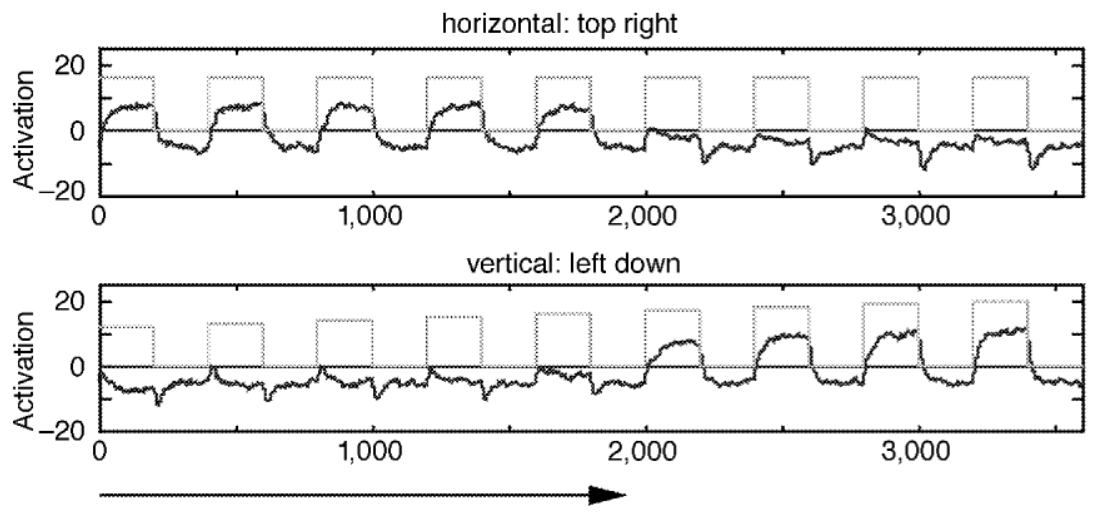

Figure 20. Simulation 7: the time-varying activation for two of the motion directions of the motion quartet when its aspect ratio is gradually decreased by decreasing the path length of vertical motion, but with the interactive coupling of all sequentially stimulated detectors set to zero (as in Simulation 5). Hysteresis effects are lost; switching occurs as soon as the aspect ratio reverses from favoring horizontal motion to favoring vertical motion. This is shown for two levels of noise strength: (A) $N=0.7$ and (B) $N=0.2$.

straint is so strong in minimal mapping theory, as well as in Dawson's (1991) connectionist version, neither can account for its reversal as a result of hysteresis.

Simulation 7: Hysteresis requires state dependence. In this simulation, the interactions among motion detectors that are stimulated during successive frame changes are removed. For simulated trials with descending aspect ratios, the elimination of these interactions is sufficient to eliminate hysteresis effects; switching occurs when the relative stimulation of horizontal and vertical detectors reverses. Vertical motion and horizontal motion are perceived equally often at the point of reversal, as is shown for two noise levels in Figure 20.

It can be concluded that interactions among successively stimulated detectors, which are the means through which perception is state dependent (see Simulation 5), are necessary in order to obtain hysteresis effects. That is, when a sequence of stimuli is presented, hysteresis occurs because pattern formation for each stimulus is strongly affected by the immediately preceding activation state of the stimulated motion detector network.

Simulation 8: Selective adaptation. Continuity in perception is adversely affected by adaptation, particularly when there is much more stimulation and, therefore, much more adaptation of the perceived than of the unperceived motion directions (i.e., when adaptation is highly selective for the motion perceived during adaptation). This is illustrated in the first part of this simulation (Figure 21A), which shows much greater adaptation of the perceived horizontal motion direction than of the unperceived vertical motion direction, as well as the discontinuity in perception that is likely as a result of the asymmerical adaptation. That is, it is probable that there will be a switch from the perception of horizontal motion to the perception of vertical motion when the adapting stimulus is replaced by a neutral test stimulus. 


\section{SIMULATION 8}

\section{(A) Selective Adaptation With More Stimulation of Horizontal Than Vertical Motion}
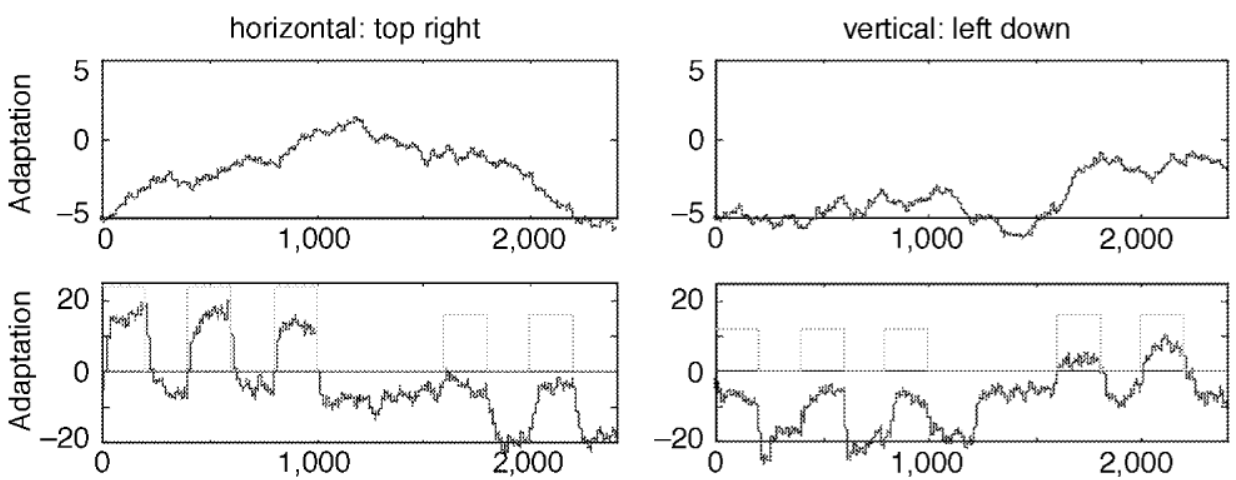

(B) Selective Adaptation With Similar Stimulation of Horizontal and Vertical Motion
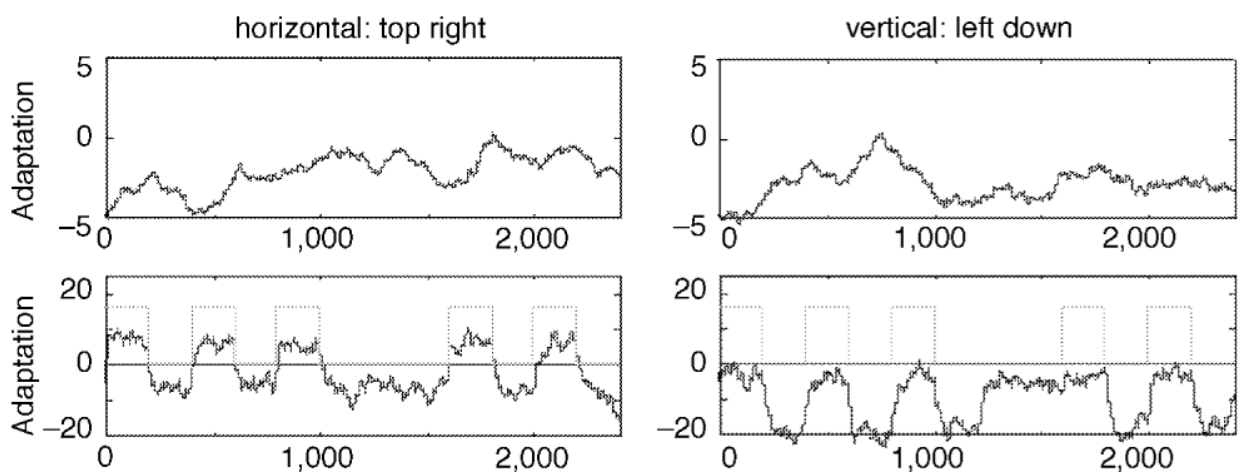

Figure 21. Simulation 8: The time course of adaptation and the time-varying activation for two of the motion directions of the motion quartet. (A) When there is more stimulation of horizontal $\left(S_{\mathrm{h}}=24\right)$ than of vertical $\left(S_{\mathrm{v}}=16\right)$ motion during the adaptation phase, adaptation is much greater for the perceived horizontal than for the unperceived vertical motion. Following a blank interval, the large asymmetry in adaptation typically results in switching to vertical motion during the test phase. (B) When there is equal stimulation of horizontal $\left(S_{\mathrm{h}}=20\right)$ and vertical $\left(S_{\mathrm{v}}=20\right)$ motion during the adaptation phase, adaptation is similar for the perceived horizontal and unperceived vertical motion. Following a blank interval, the relatively symmetrical adaptation frequently results in the continued perception of horizontal motion during the test phase.

When horizontal and vertical motion are equally stimulated, adaptation is less selective to the perceived horizontal motion than in the above case. This is illustrated in the second part of this simulation (Figure 21B), which shows similar adaptation for the perceived horizontal and the unperceived vertical motion directions, as well as the continuity in perception that is more likely as a result of less asymmetrical adaptation. That is, it is less probable that there will be a switch from the perception of horizontal motion to the perception of vertical motion when the adapting stimulus is replaced by a neutral test stimulus.

\section{Generalization and Predictions}

The simulations that follow show that the dynamical representation for the motion quartet generalizes to the motion triplet and lead to a unique prediction regarding the simultaneous perception of horizontal and vertical motion. The dynamical representation also generalizes to a monostable translational motion stimulus and leads to a unique prediction in which motions thought to be impossible are perceived.

Simulation 9: The motion triplet. It was shown in Simulation 4 that interactions among simultaneously stimulated detectors are necessary to solve the motion correspondence problem for the motion quartet. The effect of those interactions is to push the activation of all the stimulated horizontal motion detectors or all the stimulated vertical detectors below the threshold level required for perception. When the visual element in one corner of the motion quartet is removed, motions that would start or end at that location are eliminated. The four remaining motions for the motion triplet are illustrated in Figure 22A.

The critical case occurs when one motion path (e.g., vertical) is much longer than the other. On the basis of minimal mapping theory, distance-determined affinities for the triplet would be sufficiently different for horizontal motion to be perceived on the basis of the nearest neighbor constraint. For the dynamical representation, 


\section{SIMULATION 9}

(A) Frame 1

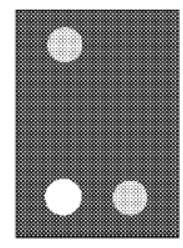

(B)

\section{Motion Triplet}

Frame 2

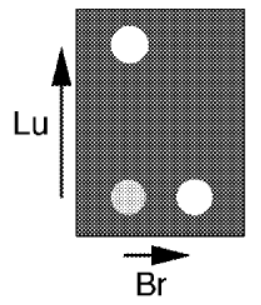

Frame 3

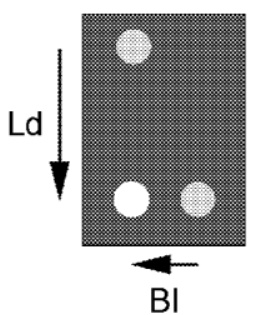

Activation for the Motion Triplet

horizontal: bottom right

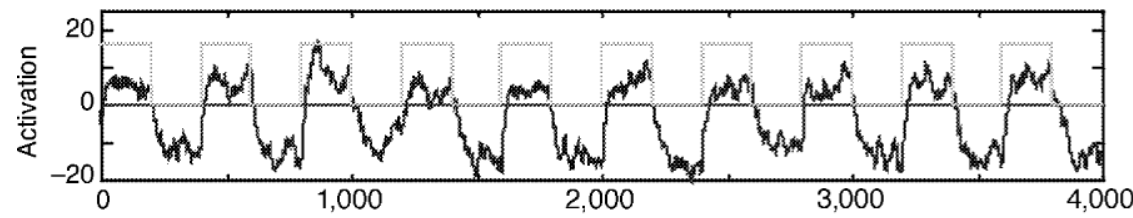

vertical: left up

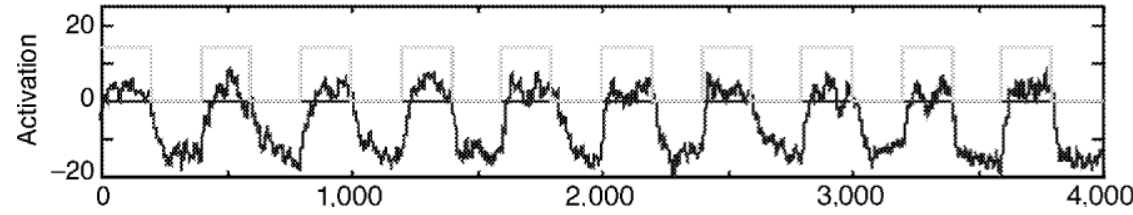

Figure 22. Simulation 9: (A) three frames of the motion triplet stimulus that is the basis for this simulation. (B) the time-varying activation for two of the motion directions of the motion triplet. Both horizontal and vertical detectors have above-threshold activation when they are stimulated, leading to the prediction (inconsistent with the nearest neighbor solution) that horizontal and vertical motion can be perceived simultaneously for the motion triplet.

eliminating motions starting or ending at the upper-right corner of the quartet eliminates their potential inhibitory influences on other stimulated detectors. As can be seen for Simulation 9 (Figure 22B), this results in the simultaneous above-threshold activation of both horizontal and vertical motion (all parameters other than the stimulus strength for vertical motion are the same as those for the motion quartet, Simulation 1). The simultaneous perception of horizontal and vertical motion is predicted by the dynamical simulation, even though the activation levels for the two motion directions are different.

Contrary to minimal mapping theory and consistent with the prediction from the dynamical representation of the motion triplet, Gilroy et al. (2001) have found that both horizontal motion and vertical motion are perceived simultaneously for the motion triplet, even when detector activation is stronger for horizontal motion because its motion path is substantially shorter than the vertical motion path. It is noteworthy that (1) the difference in path length for the motion triplet was sufficient to result in the monostable perception of horizontal motion for the motion quartet and (2) the simultaneous perception of horizontal and vertical motion for the motion triplet was obtained with a tech- nique in which Ullman's (1979) cover principle (i.e., an element cannot appear and disappear) was not a factor. ${ }^{6}$

Simulation 10: The monostable perception of translational motion. The accurate perception of motion direction for a translating rod depends on the perceived motion of its end points. Otherwise, the aperture problem arises, and motion is perceived in the direction orthogonal to the orientation of the rod (Adelson \& Movshon, 1982; Wallach, 1976). The perception of the motion paths for the end points of a horizontally translating rod is the basis for this simulation. The motion correspondence problem remains to be solved because the diagonal motion paths illustrated in Figure 13B are logically possible.

The purpose of this simulation was to determine whether the dynamical representation developed for the motion quartet also solves the motion correspondence problem for the translating rod stimulus. Some of the motion directions for this stimulus are different from the motion quartet (diagonal motions replace vertical motions), so somewhat stronger interaction strengths are introduced into the simulation for motion directions that differ by $45^{\circ}$ than for motion directions that differ by $90^{\circ}$. This was based on Marshak and Sekuler's (1979) evidence involving the 
SIMULATION 10

(A)

Translational Stimulus

Horizontal: top-right

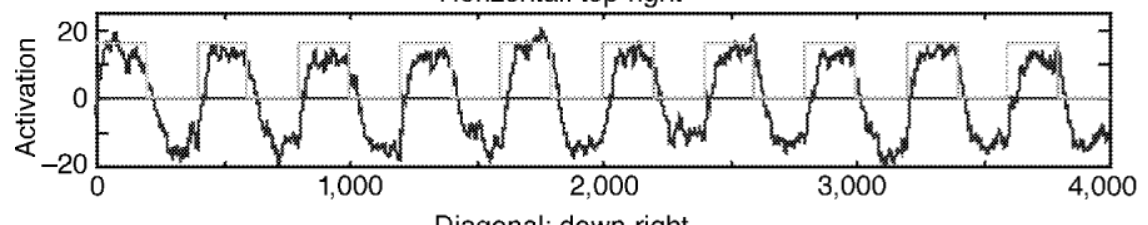

Diagonal: down-right

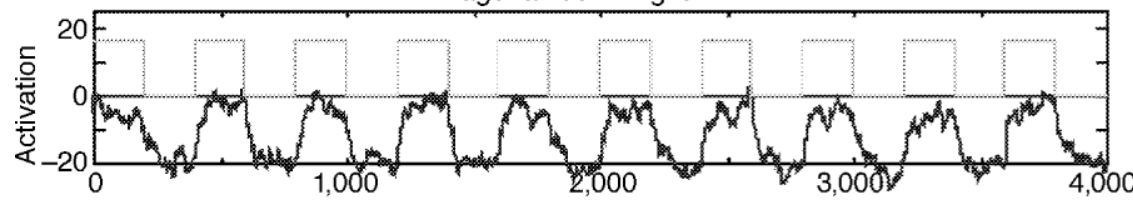

SIMULATION 11

(B) Translational Stimulus With Activational Boost to Diagonal Motions

Horizontal: top-right

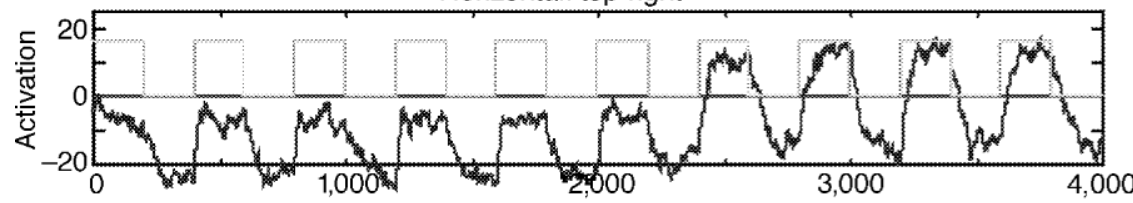

Diagonal: down-right

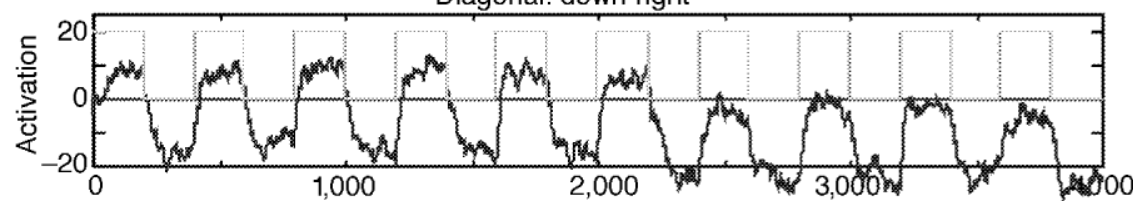

Figure 23. (A) Simulation 10: The time-varying activation for two of the motion directions of the translating rod stimulus, as illustrated in Figure 13B. The perception of horizontal motion always is signified. (B) Simulation 11: the time-varying activation for two motion directions of the translating rod stimulus when luminance bars increase the activation of diagonal motions, as illustrated in Figure $13 \mathrm{C}\left(S_{\mathrm{d}}=20\right.$, as compared with 14 in Simulation 10). Bistable diagonal and horizontal motion perception is signified.

"repulsion" of perceived motion direction. In addition, longer path lengths for the diagonal than for the horizontal motions are accounted for by weaker motion strengths for the diagonal motions (Gilroy et al., 2001).

As is illustrated in Simulation 10 (Figure 23A), the perception of horizontal translational motion is always signified (the stimulus is monostable). The reason is that horizontal motions in the same direction at the top and the bottom of the stimulus are simultaneously stimulated, so they have mutually excitatory effects on each other's activation, and in addition, the crossing diagonal motions are simultaneously stimulated, so they strongly inhibit each other. The same dynamical representation therefore can account for pattern formation for both the bistable motion quartet and the monostable translational stimulus. Differences in what the motion patterns look like for these stimuli largely stem from differences in which motion directions are stimulated simultaneously and which are stimulated sequentially.

Simulation 11: Making the translational stimulus bistable. The fact that diagonal motions are never per- ceived for the translating rod stimulus could be taken as indicative of a strong constraint against perceiving them (Kolers, 1972). However, it can be predicted from the dynamical representation that the perception of diagonal motions would become possible if the stimulus-initiated activation was sufficiently boosted for the diagonal motions, as compared with the horizontal motions. This is demonstrated in Simulation 11, which differs from Simulation 10 only with respect to the increased stimulusinitiated activation of the diagonal motions (Figure 23B). Switching from diagonal to horizontal motion occurs in the simulated trial after the sixth pair of frame changes, signifying perceptual bistability.

The means for testing this prediction come from Gilroy et al.'s (2001) evidence that motion detector activation can be increased by placing a brighter-than-background luminance bar between the start and the end of the motion path (Figure 13C). Consistent with this prediction, either diagonal or horizontal motion is perceived when this is done for the translational stimulus, with the same kind of switching behavior as that predicted by Simulation 11 . 


\section{GENERAL DISCUSSION}

The dynamical model and simulations in this article and in Giese (1999) are based on just a few, very general neural principles: (1) Detectors respond selectively to particular stimulus attributes at particular locations on the retina, as determined by their receptive field properties; (2) detector activation is stabilized by intrinsic neural mechanisms; (3) detectors are interconnected; they can enhance each other's activation through activation-dependentexcitatory interactions and/or diminish each other's activation through activation-dependentinhibitory interactions; (4) detectors are subject to random influences on their activation level; and (5) adaptation reduces a detector's activation in proportion to its current level of activation.

It has been shown in this article that when these principles are embedded in a dynamical representation, they can account for pattern formation, pattern stability, continuity in perception, and selective adaptation for the motion quartet. In addition, Giese (1999) has shown that, in simplified form, the same principles can be the basis for quantitative simulations of the results of psychophysical experiments based on the motion quartet. Although all these phenomena could potentially have been comprehensively addressed by earlier dynamical models (e.g., Bartsch \& van Hemmen, 1997; Carmesin \& Arndt, 1996; Ditzinger \& Haken, 1995; Francis \& Grossberg, 1996; Grossberg \& Mingolla, 1985; Grossberg \& Rudd, 1989, 1992; Kawamoto \& Anderson, 1985; Koechlin et al., 1999; Nowlan \& Sejnowski, 1995; Williams et al., 1986), this is the first time that it has in fact been done. The results of the dynamical simulations in this article have been contrasted with Ullman's (1979) minimal mapping theory for explaining how the visual system solves the motion correspondence problem. It was demonstrated that because random noise and stabilization mechanisms are not a part of Ullman's theory (or Dawson's, 1991, connectionist version), it cannot account for either horizontal or vertical motion being perceived for the motion quartet (but not both simultaneously) when their when their path-length-dependent affinities are matched. Nor can it account for noiseinduced violations of the nearest neighbor principle when their path-length dependent affinities are not matched. It was further demonstrated that interactions between successively stimulated detectors provide the state dependence that is the basis for continuity in perception despite gaps in time during which a motion is not stimulated and despite changes in stimulation that favor a competing motion percept. These "future-shaping" interactions, which are not a part of Ullman's theory (or Dawson's connectionist version), account for hysteresis-induced violations of the nearest neighbor principle. Finally, the simultaneous perception of horizontal and vertical motion for the motion triplet, despite large differences in path-lengthdetermined affinities, can be accounted for by the same dynamical representation (although lacking the motions that were not stimulated) that was the basis for the motion quartet simulations. It incorrectly followed from minimal mapping theory that only motion over the shorter path would be perceived for the motion triplet.

More generally, the reported simulations show that the perceived motion pattern can be embodied in the distribution of activation over a population of low-level, directionally selective motion detectors. At least for the stimuli studied thus far, neither a decision process in which the "costs" of alternative correspondence solutions are minimized (Ullman, 1979) nor higher order pattern detectors are necessary to account for motion pattern formation. Solutions to the motion correspondence problem are inherent in most dynamical theories, but in none of them is the functional significance of activation-stabilizing mechanisms made explicit, and in none are interactions between simultaneously and successively stimulated detectors distinguished with respect to their functional significance for pattern formation and pattern stability.

\section{Conclusion: The Process of Pattern Formation}

In conclusion, the theory and simulations presented in this article provide an account of motion pattern formation that is potentially generalizable to a wide range of visual stimuli. Accordingly, pattern formation begins with the presence of a stimulus that potentially stimulates a number of different detectors. As activation rises for each detector, a number of different influences on its activation are encountered: (1) intrinsic stabilization mechanisms that determine the rate at which activation increases toward its stimulus-determined value, (2) residual inhibition from prior adaptation, (3) residual interaction from previously stimulated detectors (the vehicle for state dependence), and (4) interactions from simultaneously stimulated detectors. All these influences are felt, and the motion correspondence problem solved, through their effect on the relative activation of the stimulated detectors, before the activation of any of the stimulated detectors crosses the threshold level required for perception. These solutions were shown to apply to monostable as well as to bistable pattern formation. In either case, stabilization mechanisms that respond quickly to noise-induced fluctuations in activation are necessary for coherent motion perception, suggesting that the prevention of noise-induced incoherence, not just the impossibility of bistability, is why everyday experience is so stable.

Finally, the dynamical framework described in this article for the motion quartet was shown to generalize to pattern formation for other stimuli. This was shown for both the motion triplet and the translating rod stimulus (the latter with and without the luminance bars that foster the perception of crossing motion). Although there was a large number of parameters in the simulations, a wide range of phenomena were addressed, each parameter was linked to an identifiable, experimentally relevant parameter (e.g., stimulus strength, interaction strength, noise level), and parameter values were held constant across a number of different simulations. Moreover, the results of the simulations were not highly dependent on the specific parameter values that were chosen. Currently in progress is an ex- 
tension of the dynamical approach to the counterphase row of dots, a directionally ambiguous apparent motion stimulus for which motion pattern formation is selforganized, ruling out the detection of higher order patternspecifying features as the basis for pattern perception (Hock \& Balz, 1994; Hock, Park, \& Schöner, 2001).

\section{REFERENCES}

Adelson, E. H., \& Movshon, J. A. (1982). Phenomenal coherence of moving visual patterns. Nature, 300, 523-525.

Allman, J., Miezin, F., \& McGuinness, E. (1985). Direction- and velocity-specific responses from beyond the classical receptive field in the middle temporal visual area (MT). Perception, 14, 105-126.

Attneave, F. (1974). Apparent movement and the what-where connection. Psychologia, 17, 108-120.

Bartsch, A., \& VAN Hemmen, J. L. (1997). A neural model of stroboscopic alternative motion. Network, 8, 107-126.

Braitenberg, V. (1978). Cortical architechtonics: General and areal. In M. A. B. Brazier \& H. Petsche (Eds.), Architechtonics of the cerebral cortex (IBRO MonographSeries, Vol. 3, pp. 443-466). New York: Raven.

Burt, P., \& Sperling, G. (1981). Time, distance, and feature trade-offs in visual apparent motion. Psychological Review, 88, 171-195.

CARmesin, H. O., \& ARndT, S. (1996). A neural network model for stroboscopic alternative motion. Biological Cybernetics, 75, 239-251.

Chang, J. J., \& Julesz, B. (1984). Cooperative phenomena in apparent movement perception of random-dot cinematograms. Vision Research, 24, 1781-1788.

Chaudhuri, A., \& Glaser, D. A. (1991). Metastable motion anistropy. Visual Neuroscience, 7, 397-407.

Chey, J. S., Grossberg, S., \& Mingolla, E. (1997). Neural dynamics of motion grouping: From aperture ambiguity to object speed and direction. Journal of the Optical Society of America A, 14, 2570-2594.

Dawson, M. R. W. (1991). The how and why of what went where in apparent motion: Modelling solutions to the motion correspondence problem. Psychological Review, 98, 569-603.

Deadwyler, S. A., \& Hampson, R. E. (1995). Ensemble activity and behavior: What's the code? Science, 270, 1316-1318.

Ditzinger, T., \& HAKEn, H. (1995). A synergetic model of multistability in perception. In P. Kruse \& M. Stadler (Eds.), Ambiguity in mind and nature (pp. 255-274). Berlin: Springer-Verlag.

Francis, G., \& Grossberg, S. (1996). Cortical dynamics of form and motion integration: Persistence, apparent motion, and illusory contours. Vision Research, 36, 149-173.

Giaschi, S., Douglas, R. M., Marlin, S. G., \& Cynander, M. S. (1993). The time course of direction-specific adaptation in simple and complex cells in cat striate cortex. Journal of Neurophysiology, 70, 2024-2034.

Gibson, J. J. (1966). The senses considered as perceptual systems. New York: Houghton Mifflin.

GIESE, M. A. (1999). Dynamic neural field theory of motion perception. Dordrecht: Kluwer.

Gilbert, C. D., \& Wiesel, T. N. (1990). The influence of contextual stimuli on the orientation selectivity of cells in the primary visual cortex of the cat. Vision Research, 30,1689-1701.

Gilroy, L. A., Hock, H. S., \& Ploeger, A. (2001). Differential activation solution to the motion correspondence problem. Perception \& Psychophysics, 63, 847-861.

Green, D. M., \& Swets, J. A. (1966). Signal detection theory and psychophysics. New York: Wiley.

GrossberG, S. (1973). Contour enhancement, short term memory, and constancies in reverberating neural networks. Studies in Applied Mathematics, 52, 213-257.

Grossberg, S., \& Mingolla, E. (1985). Neural dynamics of motion perception: Direction fields, apertures, and resonant grouping. Psychological Review, 92, 173-211.

Grossberg, S., \& RudD, M. E. (1989). A neuronal architecture for visual motion perception: Group and element apparent motion. Neural Networks, 2, 421-450.
Grossberg, S., \& RudD, M. E. (1992). Cortical dynamics of visual motion perception: Short-range and long-range apparent motion. Psychological Review, 99, 78-121.

Невв, D. O. (1949). The organization of behavior. New York: Wiley. Hock, H. S., \& BALz, G. W. (1994). Spatial scale dependent in-phase and anti-phase directional biases in the perception of self-organized motion patterns. Vision Research, 34, 1843-1861.

Hock, H. S., Kelso, J. A. S., \& SchöNER, G. (1993). Bistability and hysteresis in the organization of apparent motion patterns. Journal of Experimental Psychology: Human Perception \& Performance, 19, 63-80.

Hock, H. S., Kogan, K., \& Espinoza, J. K. (1997). Dynamic, statedependent thresholds for the perception of single-element apparent motion: Bistability from local cooperativity. Perception \& Psychophysics, 59, 1077-1088.

Hock, H. S., PArk, C. L., \& Schöner, G. (2001, November). A dynamical model for self-organized motion pattern formation. Paper presented at the 42nd Annual Meeting of the Psychonomic Society, Orlando, FL.

Hock, H. S., SchöNer, G., \& Hochstein, S. (1996). Perceptual stability and the selective adaptation of perceived and unperceived motion. Vision Research, 36, 3311-3323.

Hock, H. S., Schöner, G., \& Voss, A. (1997). The influence of adaptation and stochastic fluctuations on spontaneous perceptual changes for bistable stimuli. Perception \& Psychophysics, 59, 509-522.

HoEth, F. (1968). Bevorzugte Richtungen bei stroboskopischen Alternativbewegungen [Preferred directions in stroboscopic alternative motion]. Psychologische Beiträge, 16, 494-527.

JAMES, W. (1890). The principles of psychology. New York: Holt.

Kawamoto, A. H., \& Anderson, J. A. (1985). A neural network model of multistable perception. Acta Psychologia, 59, 35-65.

KIsvárdaY,Z F., Tóth, E., RAUSCH, M., \& EySEL, U. (1997). Orientationspecific relationship between populations of excitatory and inhibitory lateral connections in the visual cortex of the cat. Cerebral Cortex, 7, 605-618.

Косн, C. (1999). Biophysics of computation. New York: Oxford University Press.

Koch, C., \& Poggio, T. (1987). Biophysics of computation: Neurons, synapses, and membranes. In G. M. Edelman, W. E. Gall, \& W. M. Cowan (Eds.), Synaptic function (pp. 637-697). New York: Wiley.

Koechlin, E., Anton, J. L., \& Burnod, Y. (1999). Bayesian inference in populations of cortical neurons: A model of motion integration and segmentation in area MT. Biological Cybernetics, 80, 25-44.

KöHLER, W. (1938). Physical Gestalten. Reprinted in W. D. Ellis (Ed.), A source book of Gestalt psychology (pp. 17-54). London: Routledge $\&$ Kegan. (Original work published 1920)

KöHLER, W., \& WALlach, H. (1944). Figural after-effects: An investigation of visual processes. Proceedings of the American Philosophical Society, 88, 269-357.

Kolers, P. A. (1972). Aspects of motion perception. Oxford: Pergamon.

Kruse, P., Stadler, M., \& Wehner, T. (1986). Direction and frequency specific processing in the perception of long-range apparent movement. Vision Research, 26, 327-335.

LEVAY, S. (1988). The patchy intrinsic projections of visual cortex. Progress in Brain Research, 75, 147-161.

Levinson, E., \& SEKuler, R. (1975). The independence of channels in human vision selective for direction of movement. Journal of Physiology, 250, 347-366.

MarshaK, W., \& SeKUler, R. (1979). Mutual repulsion between moving visual targets. Science, 205, 1399-1401.

Nawrot, M., \& SeKuler, R. (1990). Assimilation and contrast in motion perception: Explorations in cooperativity. Vision Research, 30, 1439-1451.

Nowlan, S. J., \& SejnowsKI, T. J. (1995). A selection model for motion processing in area (MT) of primates. Journal of Neuroscience, 15, 1195-1214.

Petersik, J. T. (1990). Global cooperativity of the short-range process in apparent movement: Evidence obtained with contour-containing stimuli. Perception \& Psychophysics, 47, 360-368.

Ramachandran, V. S., \& Anstis, S. M. (1985). Perceptual organization in multistable apparent motion. Perception, 14, 135-143.

SCHILler, P. H. (1996). On the specificity of neurons and visual areas. Behavioural Brain Research, 76, 21-35. 
Schiller, P. von (1933). Stroboskopische Alternativversuche. Psychologische Forschung, 17, 179-214.

SCHÖNER, G., \& Hock, H. S. (1995). Concepts for a dynamical theory of perceptual organization: An example from apparent movement. In P. Kruse \& M. Stadler (Eds.), Ambiguity in mind and nature (pp. 275310). Berlin: Springer-Verlag.

Shechter, S., Hochstein, S., \& Hillman, P. (1988). Shape similarity and distance disparity as apparent motion correspondence cues. $\mathrm{Vi}$ sion Research, 29, 1013-1021.

SNOWDEN, R. J. (1989). Motions in orthogonal directions are mutually suppressive. Journal of the Optical Society of America A, 6, 10961101.

SoftKy, W. R., \& Koch, C. (1993). The highly irregular firing of cortical cells is inconsistent with temporal integration of random ESPSs. Journal of Neuroscience, 13, 334-350.

Spitz, S., \& Lipman, L. (1962). Some factors affecting Necker cube reversal rate. Perceptual \& Motor Skills, 15, 611-625.

TAnaKa, K., Fukada, Y., \& SaIto, H.-A. (1989). Underlying mechanisms of the response specificity of expansion/contraction and rotation cells in the dorsal part of the medial superior temporal area of the macaque monkey. Journal of Neurophysiology, 62, 642-656.

Thiele, A., Dobkins, K. R. \& Albright, T. D. (2000). Neural correlates of contrast detection at threshold. Neuron, 26, 715-724.

Ts'o, D. Y., Gilbert, C. D., \& Wiesel, T. N. (1986). Relationships between horizontal interactions and functional architecture in cat striate cortex as revealed by cross-correlation analysis. Journal of Neuroscience, 6, 1160-1170.

Ullman, S. (1979). The interpretation of visual motion. Cambridge, MA: MIT Press.

WALLACH, H. (1976). The direction of motion of straight lines. In H. Wallach (Ed.), On perception (pp. 201-216). New York: New York Times Book Co., Quadrangle Books.

Williams, D., Phillips, G., \& Sekuler, R. (1986). Hysteresis in the perception of motion direction as evidence for neural cooperativity. Nature, 324, 253-255.

Williams, D., \& SeKuler, R. (1984). Coherent global motion percepts from stochastic local motions. Vision Research, 24, 55-62.

WiLson, H. R. (1998). Spikes, decisions, and actions: Dynamical foundations of neuroscience. Oxford: Oxford University Press.

Wilson, H. R, Ferrera, V. P., \& Yo, C. (1992). A psychophysically motivated model for two-dimensional motion perception. Visual Neuroscience, 9, 79-97.

Wilson, H. R., \& KIM, J. (1994). A model for motion coherence and transparency. Visual Neuroscience, 11, 1205-1220.

\section{NOTES}

1. Hock et al. (1996) adapted subjects to motion quartets for which horizontal motion was perceived when the vertical motion path was relatively short (aspect ratio $=1.25$ ) and when the vertical motion path was relatively long (aspect ratio $=2.25$ ). They tested for the effects of adap- tation with motion trapezoid configurations that were either horizontally oriented (so that oblique and horizontal motion competed) or vertically oriented (so that oblique and vertical motion competed). Adaptation to the perceived horizontal motion was indicated by the perception of more oblique motion for the horizontally oriented trapezoid for the larger adapting aspect ratio. Adaptation to the unperceived vertical motion was indicated by the perception of more oblique motion for the vertically oriented trapezoid for the smaller adapting aspect ratio.

2. Hock, Schöner, and Voss (1997) varied the duration of trials for a motion quartet with an aspect ratio of one and found that when horizontal motion was the initial percept, the probability of at least one switch per trial increased with trial duration. This increase followed a temporal independence function calculated on the basis of the probability of a switch during a pair of frames remaining constant over the course of every trial (differential adaptation would have increased this probability as a function of trial duration).

3 . This article focuses on the activation of individual detectors that respond selectively to the attributes of visual stimuli. However, when even a small local stimulus is presented, the overlap of receptive fields for different detecting units results in a finite number being activated simultaneously, with different local ensembles (first called cell assemblies by Hebb, 1949) being activated to isolate the detectors' response to individual stimulus dimensions (e.g., Deadwyler \& Hampson, 1995; P. H. Schiller, 1996). The local ensemble would presumably "inherit" the noise-stabilizing mechanisms intrinsic to each of its members. For the purposes of this article, the terms detector and detector ensemble are interchangeable.

4. Evidence for inhibitory competition between motions in orthogonal as well as opposite directions has been reported by many investigators (e.g., Levinson \& Sekuler, 1975; Snowden, 1989; Thiele, Dobkins, $\&$ Albright, 2000), so they are a necessary part of the dynamical representation. Evidence for excitatory interactions among motion detectors with the same directional selectivity comes from studies with random cinematograms (e.g., Chang \& Julesz, 1984; Nawrot \& Sekuler, 1990; Petersik, 1990; Williams \& Sekuler, 1984) and directionally ambiguous rows of dots (Hock \& Balz, 1994).

5. Hock et al. (1993) introduced a modification of the method of limits that eliminates response persistence as a source of hysteresis effects. Trials with ascending or descending sequences of aspect ratios varied with respect to their end-point aspect ratio, and participants indicated without speed stress whether there was a switch from an initially established percept at any time during the trial. When switches occurred was determined by how deeply trials receiving switch responses penetrated the range of aspect ratios for which perception for the motion quartet is bistable.

6. Gilroy et al.'s (2001) motion triplets were based on the generalized version of apparent motion (Hock, Kogan, \& Espinoza, 1997), for which all element locations are simultaneously visible, although with different luminance values. As is illustrated in Figure 22A, motion is perceived when the luminance values are exchanged during successive frames. Because an element always was present at each corner of the triplet, the constraint of the cover principle did not apply. 


\section{APPENDIX}

The dynamical model of motion perception for the motion quartet (Figure 1B) invokes the eight possible motions illustrated in Figure 13A, each represented by an activation variable, $u_{i}(i=\mathrm{Br}, \mathrm{Bl}, \mathrm{Tr}, \mathrm{Tl}, \mathrm{Ru}, \mathrm{Rd}, \mathrm{Lu}, \mathrm{Ld})$. The dynamics of each activation variable sums the contribution of the intrinsic stabilization mechanism $\left(-u_{i}\right)$, additive inputs [resting level, $h$, and stimulation, $S_{i}(t)$ ], Gaussian white noise, $\xi_{i}(t)$, as well as interactions from the seven other activation variables. Interactions are mediated by the sigmoidal nonlinearity

$$
\sigma(u)=\frac{1}{1+\exp (-\beta u)} .
$$

The interaction strengths, $w_{\phi}$, depend on the angle, $\phi$, between the motion directions. The complete set of dynamical equations is as follows.

$$
\begin{aligned}
\tau \dot{u}_{\mathrm{Br}}= & -u_{\mathrm{Br}}+h+S_{\mathrm{Br}}(t)+q \xi_{\mathrm{Br}}(t) \\
& +w_{0} \sigma\left(u_{\mathrm{Tr}}\right)-w_{180} \sigma\left(u_{\mathrm{Tl}}\right)-w_{180} \sigma\left(u_{\mathrm{Bl}}\right) \\
& -w_{90} \sigma\left(u_{\mathrm{Lu}}\right)-w_{90} \sigma\left(u_{\mathrm{Ld}}\right)-w_{90} \sigma\left(u_{\mathrm{Rd}}\right)-w_{90} \sigma\left(u_{\mathrm{Ru}}\right) . \\
\tau \dot{u}_{\mathrm{Bl}}= & -u_{\mathrm{Bl}}+h+S_{\mathrm{Bl}}(t)+q \xi_{\mathrm{Bl}}(t) \\
& +w_{0} \sigma\left(u_{\mathrm{Tl}}\right)-w_{180} \sigma\left(u_{\mathrm{Tr}}\right)-w_{180} \sigma\left(u_{\mathrm{Br}}\right) \\
& -w_{90} \sigma\left(u_{\mathrm{Ru}}\right)-w_{90} \sigma\left(u_{\mathrm{Rd}}\right)-w_{90} \sigma\left(u_{\mathrm{Ld}}\right)-w_{90} \sigma\left(u_{\mathrm{Lu}}\right) . \\
\tau \dot{u}_{\mathrm{Tr}}= & -u_{\mathrm{Tr}}+h+S_{\mathrm{Tr}}(t)+q \xi_{\mathrm{Tr}}(t) \\
& +w_{0} \sigma\left(u_{\mathrm{Br}}\right)-w_{180} \sigma\left(u_{\mathrm{Bl}}\right)-w_{180} \sigma\left(u_{\mathrm{Tl}}\right) \\
& -w_{90} \sigma\left(u_{\mathrm{Ld}}\right)-w_{90} \sigma\left(u_{\mathrm{Lu}}\right)-w_{90} \sigma\left(u_{\mathrm{Ru}}\right)-w_{90} \sigma\left(u_{\mathrm{Rd}}\right) . \\
\tau \dot{u}_{\mathrm{Tl}}= & -u_{\mathrm{Tl}}+h+S_{\mathrm{Tl}}(t)+q \xi_{\mathrm{Tl}}(t) \\
& +w_{0} \sigma\left(u_{\mathrm{B} 1}\right)-w_{180} \sigma\left(u_{\mathrm{Br}}\right)-w_{180} \sigma\left(u_{\mathrm{Tr}}\right) \\
& -w_{90} \sigma\left(u_{\mathrm{Rd}}\right)-w_{90} \sigma\left(u_{\mathrm{Ru}}\right)-w_{90} \sigma\left(u_{\mathrm{Lu}}\right)-w_{90} \sigma\left(u_{\mathrm{Ld}}\right) . \\
\tau \dot{u}_{\mathrm{Ru}}= & -u_{\mathrm{Ru}}+h+S_{\mathrm{Ru}}(t)+q \xi_{\mathrm{Ru}}(t) \\
& +w_{0} \sigma\left(u_{\mathrm{Lu}}\right)-w_{180} \sigma\left(u_{\mathrm{Ld}}\right)-w_{180} \sigma\left(u_{\mathrm{Rd}}\right) \\
& -w_{90} \sigma\left(u_{\mathrm{Bl}}\right)-w_{90} \sigma\left(u_{\mathrm{Br}}\right)-w_{90} \sigma\left(u_{\mathrm{Tr}}\right)-w_{90} \sigma\left(u_{\mathrm{T} 1}\right) . \\
\tau \dot{u}_{\mathrm{Rd}}= & -u_{\mathrm{Rd}}+h+S_{\mathrm{Rd}}(t)+q \xi_{\mathrm{Rd}}(t) \\
& +w_{0} \sigma\left(u_{\mathrm{Ld}}\right)-w_{180} \sigma\left(u_{\mathrm{Lu}}\right)-w_{180} \sigma\left(u_{\mathrm{Ru}}\right) \\
& -w_{90} \sigma\left(u_{\mathrm{Tl}}\right)-w_{90} \sigma\left(u_{\mathrm{Tr}}\right)-w_{90} \sigma\left(u_{\mathrm{Br}}\right)-w_{90} \sigma\left(u_{\mathrm{B} 1}\right) . \\
\tau \dot{u}_{\mathrm{Lu}}= & -u_{\mathrm{Lu}}+h+S_{\mathrm{Lu}}(t)+q \xi_{\mathrm{Lu}}(t) \\
& +w_{0} \sigma\left(u_{\mathrm{Ru}}\right)-w_{180} \sigma\left(u_{\mathrm{Rd}}\right)-w_{180} \sigma\left(u_{\mathrm{Ld}}\right) \\
& -w_{90} \sigma\left(u_{\mathrm{Br}}\right)-w_{90} \sigma\left(u_{\mathrm{Bl}}\right)-w_{90} \sigma\left(u_{\mathrm{Tl}}\right)-w_{90} \sigma\left(u_{\mathrm{Tr}}\right) . \\
\tau \dot{u}_{\mathrm{Ld}}= & -u_{\mathrm{Ld}}+h+S_{\mathrm{Ld}}(t)+q \xi_{\mathrm{Ld}}(t) \\
& +w_{0} \sigma\left(u_{\mathrm{Rd}}\right)-w_{180} \sigma\left(u_{\mathrm{Ru}}\right)-w_{180} \sigma\left(u_{\mathrm{Lu}}\right) \\
& -w_{90} \sigma\left(u_{\mathrm{Tr}}\right)-w_{90} \sigma\left(u_{\mathrm{Tl}}\right)-w_{90} \sigma\left(u_{\mathrm{Bl}}\right)-w_{90} \sigma\left(u_{\mathrm{Br}}\right) .
\end{aligned}
$$

Input functions, $S_{i}(t)$, represent the time courses of signals from motion detectors, approximatedhere as square waves that alternate on and off every $200 \mathrm{msec}$, as shown in the simulation figures: $S_{i}(t)=S_{0}$ following a frame change that stimulates motion $i$, and $S_{i}(t)=0$ following a frame change that does not stimulate motion $i$.

All simulations are based on one set of parameters: $\tau=20 \mathrm{msec}, h=-5, \beta=1, w_{90}=7, w_{180}=w_{0}=3.5$, $q=0.7$, and $S_{0}=16$, with the following exceptions. For Simulation 2, noise strength, $q$, was reduced to 0.07 (part A) and 0 (parts B and C). Stimulus strength was increased to $S_{0}=24$ for all horizontal and vertical directions in part C of Simulation 2. For Simulation 3, stimulus strength, $S_{0}$, was 16.5 for all horizontal directions. For Simulation 6, $S_{0}$ for vertical motion varied from 12 to 20 for descending trials and from 20 to 12 for ascending trials. For Simulation 7, $S_{0}$ varied for vertical motion from 12 to 20 for descending trials. In Simulations 4,5 , and 7 , interaction strengths were selectively set to zero, as is described in the text.

The motion triplet (Figure 22A) was simulated on the basis of the same equations (Simulation 9). Because the activation variables $u_{\mathrm{Tr}}, u_{\mathrm{Tl}}, u_{\mathrm{Ru}}$, and $u_{\mathrm{Rd}}$ were never stimulated (correspondinginputs equal zero), they did not contribute to the dynamic interactions and were omitted from the simulations. The two stimulus strengths for vertical and horizontal were 14 and 16 , respectively. 


\section{APPENDIX (Continued)}

The translating rod stimulus is modeled with the same dynamical representation but involves a different set of possible motions, as is illustrated in Figure 14B. The analogous set of equations is as follows.

$$
\begin{aligned}
& \tau \dot{u}_{\mathrm{Br}}=-u_{\mathrm{Br}}+h+S_{\mathrm{Br}}(t)+q \xi_{\mathrm{Br}}(t) \\
& +w_{0} \sigma\left(u_{\mathrm{Tr}}\right)-w_{180} \sigma\left(u_{\mathrm{Tl}}\right)-w_{180} \sigma\left(u_{\mathrm{Bl}}\right) \\
& -w_{45} \sigma\left(u_{\text {Dur }}\right)-w_{45} \sigma\left(u_{\text {Ddl }}\right)-w_{45} \sigma\left(u_{\text {Dul }}\right)-w_{45} \sigma\left(u_{\text {Ddr }}\right) \text {. } \\
& \tau \dot{u}_{\mathrm{B} 1}=-u_{\mathrm{B} 1}+h+S_{\mathrm{Bl}}(t)+q \xi_{\mathrm{Bl}}(t) \\
& +w_{0} \sigma\left(u_{\mathrm{Tl}}\right)-w_{180} \sigma\left(u_{\mathrm{Tr}}\right)-w_{180} \sigma\left(u_{\mathrm{Br}}\right) \\
& -w_{45} \sigma\left(u_{\text {Dur }}\right)-w_{45} \sigma\left(u_{\text {Ddl }}\right)-w_{45} \sigma\left(u_{\text {Dul }}\right)-w_{45} \sigma\left(u_{\text {Ddr }}\right) \text {. } \\
& \tau \dot{u}_{\mathrm{Tr}}=-u_{\mathrm{Tr}}+h+S_{\mathrm{Tr}}(t)+q \xi_{\mathrm{Tr}}(t) \\
& +w_{0} \sigma\left(u_{\mathrm{Br}}\right)-w_{180} \sigma\left(u_{\mathrm{B} 1}\right)-w_{180} \sigma\left(u_{\mathrm{Tl}}\right) \\
& -w_{45} \sigma\left(u_{\text {Dur }}\right)-w_{45} \sigma\left(u_{\text {Ddl }}\right)-w_{45} \sigma\left(u_{\text {Dul }}\right)-w_{45} \sigma\left(u_{\text {Ddr }}\right) \text {. } \\
& \tau \dot{u}_{\mathrm{Tl}}=-u_{\mathrm{T} 1}+h+S_{\mathrm{Tl}}(t)+q \xi_{\mathrm{Tl}}(t) \\
& +w_{0} \sigma\left(u_{\mathrm{B} 1}\right)-w_{180} \sigma\left(u_{\mathrm{Br}}\right)-w_{180} \sigma\left(u_{\mathrm{Tr}}\right) \\
& -w_{45} \sigma\left(u_{\text {Dur }}\right)-w_{45} \sigma\left(u_{\text {Ddl }}\right)-w_{45} \sigma\left(u_{\text {Dul }}\right)-w_{45} \sigma\left(u_{\text {Ddr }}\right) \text {. } \\
& \tau \dot{u}_{\text {Dur }}=-u_{\text {Dur }}+h+S_{\text {Dur }}(t)+q \xi_{\text {Dur }}(t) \\
& -w_{180} \sigma\left(u_{\mathrm{Ddl}}\right)-w_{90} \sigma\left(u_{\mathrm{Dul}}\right)-w_{90} \sigma\left(u_{\mathrm{Ddr}}\right) \\
& -w_{45} \sigma\left(u_{\mathrm{B} 1}\right)-w_{45} \sigma\left(u_{\mathrm{Br}}\right)-w_{45} \sigma\left(u_{\mathrm{Tr}}\right)-w_{45} \sigma\left(u_{\mathrm{T} 1}\right) \text {. } \\
& \tau \dot{u}_{\mathrm{Ddl}}=-u_{\mathrm{Ddl}}+h+S_{\mathrm{Ddl}}(t)+q \xi_{\mathrm{Ddl}}(t) \\
& -w_{180} \sigma\left(u_{\mathrm{Dur}}\right)-w_{90} \sigma\left(u_{\mathrm{Dul}}\right)-w_{90} \sigma\left(u_{\mathrm{Ddr}}\right) \\
& -w_{45} \sigma\left(u_{\mathrm{T} 1}\right)-w_{45} \sigma\left(u_{\mathrm{Tr}}\right)-w_{45} \sigma\left(u_{\mathrm{Br}}\right)-w_{45} \sigma\left(u_{\mathrm{B} 1}\right) \text {. } \\
& \tau \dot{u}_{\mathrm{Dul}}=-u_{\mathrm{Dul}}+h+S_{\mathrm{Dul}}(t)+q \xi_{\mathrm{Dul}}(t) \\
& -w_{180} \sigma\left(u_{\mathrm{Ddr}}\right)-w_{0} \sigma\left(u_{\mathrm{Dur}}\right)-w_{90} \sigma\left(u_{\mathrm{Ddl}}\right) \\
& -w_{45} \sigma\left(u_{\mathrm{Br}}\right)-w_{45} \sigma\left(u_{\mathrm{B} 1}\right)-w_{45} \sigma\left(u_{\mathrm{Tl}}\right)-w_{45} \sigma\left(u_{\mathrm{Tr}}\right) \text {. } \\
& \tau \dot{u}_{\mathrm{Ddr}}=-u_{\mathrm{Ddr}}+h+S_{\mathrm{Ddr}}(t)+q \xi_{\mathrm{Ddr}}(t) \\
& -w_{180} \sigma\left(u_{\mathrm{Dul}}\right)-w_{90} \sigma\left(u_{\mathrm{Dur}}\right)-w_{90} \sigma\left(u_{\mathrm{Ddl}}\right) \\
& -w_{45} \sigma\left(u_{\mathrm{Tr}}\right)-w_{45} \sigma\left(u_{\mathrm{Tl}}\right)-w_{45} \sigma\left(u_{\mathrm{Bl}}\right)-w_{45} \sigma\left(u_{\mathrm{Br}}\right) \text {. }
\end{aligned}
$$

The strength of the new interaction was $w_{45}=9$. For Simulation 10, diagonal motion input strength was 14 , and for Simulation 11, diagonal motion input strength was 20. All other parameter values were identical to the motion quartet.

Adaptation dynamics were introduced for Simulation 8 . For each activation variable, $u_{\mathrm{i}}$, an adaptation variable, $v_{i}$, evolves with a time constant, $\tau_{v}=1,000 \mathrm{msec}$ according to

$$
\tau_{v} v_{i}=-v_{i}+h_{v}+\Delta h \sigma\left(u_{i}\right)
$$

In the absence of positive activation, the adaptation variable relaxes to the resting level, $h_{v}=-5$. For strong positive activation, the maximum level of adaptation is $h_{v}+\Delta h=5$. The adaptation variables, in turn, act as inhibitory input to the dynamics of each activation variable, but through a sigmoidal nonlinearity, only positive levels of adaptation are effective:

$$
\tau \dot{u}_{i}=\ldots-c_{v} \sigma\left(v_{i}\right)
$$

The adaptive strength was $c_{v}=5$.

(Manuscript received May 23, 2001;

revision accepted for publication September 19, 2002.) 